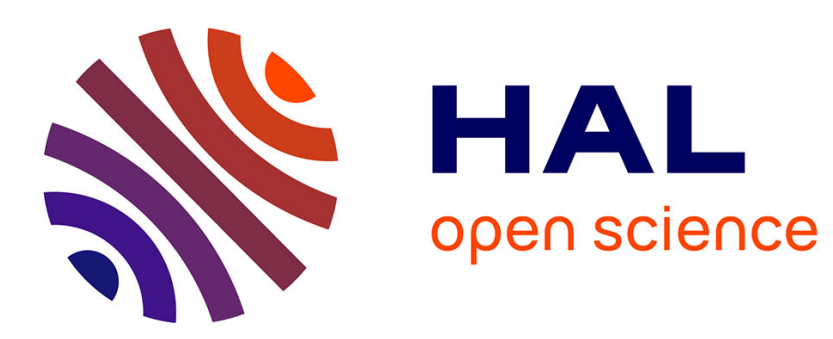

\title{
Membrane trafficking of yeast transporters: mechanisms and physiological control of downregulation
}

\author{
Rosine Haguenauer-Tsapis, Bruno André
}

\section{To cite this version:}

Rosine Haguenauer-Tsapis, Bruno André. Membrane trafficking of yeast transporters: mechanisms and physiological control of downregulation. Topics in Current Genetics, 2004, 9, pp.273-323. 10.1007/b97215 . hal-00007691

\section{HAL Id: hal-00007691 \\ https://hal.science/hal-00007691}

Submitted on 26 Jul 2005

HAL is a multi-disciplinary open access archive for the deposit and dissemination of scientific research documents, whether they are published or not. The documents may come from teaching and research institutions in France or abroad, or from public or private research centers.
L'archive ouverte pluridisciplinaire HAL, est destinée au dépôt et à la diffusion de documents scientifiques de niveau recherche, publiés ou non, émanant des établissements d'enseignement et de recherche français ou étrangers, des laboratoires publics ou privés. 


\title{
Membrane trafficking of yeast transporters: mechanisms and physiological control of downregulation
}

Haguenauer-Tsapis, Rosine\# and André, Bruno*

\#Institut Jacques Monod-CNRS, Universités Paris VI and VII, 2 place Jussieu 75251 Paris Cedex 05 France

*Laboratoire de Physiologie Cellulaire, Institut de Biologie et de Médecine Moléculaires, Université Libre de Bruxelles, P.O. Box 300, Rue des Professeurs Jeener et Brachet 12, 6041

Gosselies, BELGIUM

\begin{abstract}
:
Of the 125 plasma membrane transporters thus far identified in the yeast $S$. cerevisiae, a growing number is reported to be subject to tight control at membrane trafficking level, in addition to control at transcriptional level. Typical physiological conditions inducing these controls include changes of substrate concentration and availability of alternative nutrients. These changes of conditions often provoke the downregulation of specific transporters eventually accompanied by upregulation of others which are more appropriate for the new conditions. Downregulation generally involves the onset or acceleration of endocytosis of the transporter and subsequent targeting to the vacuole where it is degraded. In many cases, the same physiological signals also induce diversion of the neosynthesized transporter from the Golgi apparatus to the endosomal/vacuolar degradation pathway without passing through the plasma membrane. In the present review, we first describe the main factors - including the ubiquitin ligase Rsp5p and its partners - involved in downregulation of yeast transporters, at endocytosis and endosomal/vacuolar targeting steps. We then summarize the wide variety of physiological situations reported to induce the downregulation of specific yeast transporters. Finally, we discuss possible mechanisms responsible for a specific transporter to be ubiquitylated and downregulated only under particular conditions. Evidence is accumulating that these mechansisms involve association of the protein with specific lipid environments, possible phosphorylation by protein kinases, and specific binding of the transporter to its own substrates.
\end{abstract}

\section{Introduction}

About 282 transmembrane solute transporters have been inventoried in the yeast Saccharomyces cerevisiae, a minimum of 125 of which are known to be located at the plasma membrane (Van Belle and André 2001; Huh et al. 2003). Some of these transporters, such as the proton ATPase Pmalp, are essential in all growth conditions. Such proteins are continuously synthesized and are present in an active and stable form at the cell surface. There is growing evidence that these "constitutive" transporters make up only a small fraction of the yeast transportome. Indeed, the number of yeast transporters reported to be subject to tight regulation according to environmental conditions is increasing all the time. The mechanisms by which cells control transporters act essentially at three levels: gene transcription, intracellular membrane trafficking of the synthesized proteins, and modulation of intrinsic activity. As the same principles seem to govern the regulation of transporters in the cells of plants and animals, yeast constitutes a powerful model for dissecting the underlying mechanisms in detail. 
In the last ten years, a large number of studies have focused on the regulation of yeast transporters at the level of membrane trafficking. At least three types of control have been described to date. First, a widespread and efficient way of eliminating a transporter is to trigger its specific sorting into endocytic vesicles and to target the internalized protein to the endosome/vacuolar degradation pathway. Second, the fate of newly synthesized transporters within the secretory pathway is also frequently regulated, the protein being either targeted to the cell surface or diverted to the vacuole without passing through the plasma membrane. Third, some transporters may be stored in intracellular membranes and recycled to the plasma membrane in response to specific signals. Typical physiological situations inducing these control mechanisms include changes of substrate concentration, availability of alternative nutrients, or the transfer of cells to adverse conditions.

We review here current knowledge concerning the mechanisms controlling the membrane trafficking of yeast plasma membrane transporters, making reference to similarities with the membrane trafficking of mammalian transporters. As we still know little about the regulation of internal membrane transporters (about 90 proteins in yeast), this topic will not be covered here.

\section{1. Trafficking of plasma membrane transporters along the secretory pathway}

Studies of thermosensitive secretory ( $\mathrm{sec}$ ) mutants provided an early demonstration that plasma membrane transporters follow the secretory pathway to the plasma membrane, with no inducible permease activity detected after induction in sec mutants placed at restrictive temperature (first reports (Ferro-Novick et al. 1984; Tschopp et al. 1986; Jund et al. 1988) (Fig. 1). These initial reports were followed by other studies providing further insight into the trafficking of yeast transporters to the plasma membrane. However, this was not the object of intense investigations as many of the genes/proteins involved in this process are also involved in the trafficking of soluble proteins along the secretory pathway. We will briefly summarize some of the general and specific features of transporter trafficking along the secretory pathway.

\section{1. 1 ER-associated events}

Transporters are first inserted into the endoplasmic reticulum (ER) membrane. They are then targeted to the Golgi apparatus and packaged into secretory vesicles that ultimately fuse with the plasma membrane at the incipient bud. Most yeast transporters have no cleavable signal sequence. They are targeted to the ER by the first hydrophobic segment, which functions as an internal signal sequence, as has been demonstrated experimentally in a few cases (Silve et al. 1991; Kolling and Hollenberg 1994b). Transporters are thought to be co-translationally inserted into the ER membrane, acquiring their final topology. Computer-aided sequence analysis based on hydropathy profile analysis has provided useful topological predictions for transporters. However, whereas extensive studies have been carried out for bacterial and mammalian transporters, the topology of yeast transporters has received much less attention. A few studies based on protease protection (Silve et al. 1991; Kolling and Hollenberg 1994b), or glycosylation scanning experiments (Ahmad and Bussey 1988; Garnier et al. 1996; Gilstring and Ljungdahl 2000) have been reported. The only complete in vivo analysis of the topology of a yeast transporter published to date is that for the general aminoacid permease (Gap1p), which has twelve transmembrane spans and $\mathrm{N}$ - and $\mathrm{C}$-termini oriented towards the 
cytoplasm (Gilstring and Ljungdahl 2000). Most plasma membrane transporters appear to have cytoplasmic N-termini, often longer than those of their bacterial orthologs, and shorter than those of their mammalian orthologs. Exceptions include the ammonium Mep2p transporter, which has luminal/external N-terminus (Marini and André 2000). Structure/function studies have identified signals in the cytoplasmic regions of transporters in the $\mathrm{N}$ - and $\mathrm{C}$-terminal regions in particular - that are important for internalization.

The translocation of soluble proteins across the ER and the ER insertion of membranebound proteins are accompanied by the folding of these proteins. Misfolded proteins undergo ER-associated degradation (ERAD), which involves a proteolytic pathway including retrotranslocation (soluble proteins) or dislocation (membrane-bound proteins) of the cargo proteins and their concomitant ubiquitylation by cytosolic enzymes, followed by their degradation by the proteasome (Hampton 2002; Kostova and Wolf 2003). A certain point mutation $(\Delta \mathrm{F} 508)$ in the mammalian cystic fibrosis transmembrane conductance regulator $\mathrm{Cl}$ channel (CFTR) leads to ERAD of the mutant protein. This leads to a major decrease in steady-state plasma membrane levels of CFTR, the basis of the common inherited disease cystic fibrosis. The ERAD of CFTR is regarded as the prototype of ERAD of membranebound proteins and the events involved in this process have been extensively studied. The ERAD of several yeast plasma membrane proteins has also been reported, including the case of the uracil permease Fur4p (Galan et al. 1997), the ABC transporter Pdr5p (Plemper et al. 1998), the a-factor transporter Ste6p (Loayza et al. 1998), and the plasma membrane $\left(\mathrm{H}^{+}\right)$ ATPase Pma1p (Wang et al. 1999). Early studies indicated that yeast was potentially a good model system for studying the ERAD of CFTR (Paddon et al. 1996). One critical step in ERAD is the recognition, by specific ubiquitin protein ligases, of substrates that have to be ubiquitylated. A RING-finger ubiquitin ligase, Hrd1/Der3, was initially reported to be responsible for the ubiquitylation of a subset of soluble and membrane-bound ERAD substrates, but this enzyme played no part in the degradation of misfolded uracil permease, or mammalian CFTR produced in yeast (Hampton 2002). Another ER membrane-embedded ubiquitin ligase involved in the degradation of a few ERAD substrates, Doa10p, was recently identified (Swanson et al. 2001), but little is known about its function.

The transporters leave the ER once they are correctly folded. Efficient transport of membrane and secretory proteins from the ER requires concentrative and signal-mediated sorting into coat protein complex II (COPII)-coated vesicles. In the case of Gap1p, this sorting involves a C-terminal di-acidic signal (Malkus et al. 2002) recognized by the COPII protein Sec24p, that has multiple independent sites of cargo recognition (Miller et al. 2003), Correct ER exit of transporters in COPII-coated vesicles requires ER-associated proteins, which help packaging of specific class of transporters (Ljungdahl, this issue) (Fig. 1).

\section{1. 2 Posttranslational modifications}

Many soluble proteins display posttranslational modifications in their trafficking along the secretory pathway, in both the ER and Golgi apparatus (glycosylation, formation of disulfide bonds, proteolytic cleavages) (Table 1). Only a few yeast transporters have been reported to undergo glycosylation. Indeed, several yeast plasma membrane transporters, including the uracil permease Fur4p, the a-factor transporter Ste6p, or the Hxt2p high affinity glucose transporter, and a putative anion exchanger (Ynl275p), have clearly been shown not to be glycosylated (Volland et al. 1992; Kuchler et al. 1993; Wendell and Bisson 1993; Zhao and Reithmeier 2001). Unglycosylated transporters include a number of proteins with potential 
glycosylation sites (Asn/X/Ser or Thr). These sites may be cytoplasmic, within transmembrane segments, or luminal, but they are generally positioned too close to the plane of the membrane for oligosaccharyl transferases to have access (Garnier et al. 1996). The few reported examples of glycosylated transporters include the Mep2p ammonium transporter, which is N-glycosylated in its N-terminal domain (Marini and André 2000), the endosomal transporter Nhx1p (Wells and Rao 2001), or the copper transporter Ctr1p, which displays extensive O-glycosylation (Dancis et al. 1994). An apparently common posttranslational modification of many plasma membrane transporters is the phosphorylation of serine residues (Table 1 and list in (Rotin et al. 2000)). The plasma membrane $\left[\mathrm{H}^{+}\right]$ATPase, Pma1p (Chang and Slayman 1991), uracil permease (Volland et al. 1992), and the a-factor transporter Ste6p (Kuchler et al. 1993) were among the first serine-phosphorylated transporters to be identified. Experiments in sec mutants demonstrated that several residues of Pma1p are phosphorylated at different steps of the secretory pathway (Chang and Slayman 1991), whereas uracil permease is mainly phosphorylated after its arrival at the plasma membrane (Volland et al. 1992). C-terminal phosphorylation of Pma1p has been shown to be involved in the activity of this protein (Goossens et al. 2000), whereas the role played by phosphorylation remains unclear for most transporters. However, phosphorylation has been shown to control the rate of endocytosis of transporters in some cases (Marchal et al. 1998; Medintz et al. 2000; Kolling 2002).

\section{1. 3 ER-to-Golgi trafficking, and beyond}

Various studies have emphasized the critical role played by lipids in the correct trafficking of plasma membrane proteins in the late steps of the secretory pathway. Transporters are no exception (Opekarova, this issue). We will therefore focus here exclusively on certain general features of the requirement for lipids, paying particular attention to the role played by rafts in transporter trafficking. Rafts were recently described as dynamic assemblies of cholesterol and sphingolipids that form in the exoplasmic leaflet of cellular bilayer membranes (Ikonen 2001). Yeast has rafts composed of sphingolipids and of the structural plasma membrane sterol, ergosterol. They have been identified experimentally as fractions resistant to Triton X100 at $4^{\circ} \mathrm{C}$. Fur4p and Pma1p have been reported to be associated with rafts in the ER (Dupre and Haguenauer-Tsapis 2003) and the Golgi apparatus (Bagnat et al. 2001), respectively. Impairment of association with rafts due to the inhibition of sphingolipid synthesis delays the delivery of Fur4p to the plasma membrane (Dupre and Haguenauer-Tsapis 2003). The inhibition of sphingolipids or of ergosterol synthesis has been reported to lead to the routing of Pma1p (Bagnat et al. 2001) or the Tat2p tryptophan permease (Umebayashi and Nakano 2003) directly from the Golgi apparatus to the vacuole, rather than the plasma membrane. Association with rafts seems to depend upon the conformational features of nascent proteins, and mutant forms of Pma1p that fail to associate with rafts are also directly targeted from the Golgi apparatus for vacuolar degradation (Arvan et al. 2002). Thus, during the synthesis of membrane-bound proteins, quality control mechanisms may operate in both the ER and Golgi compartments.

Some of the signals important in protein trafficking in yeast, such as di-lysine, which governs the retrieval of membrane proteins from the Golgi back to the ER, or acidic cluster signals, which are involved in recycling from endosomes back to the Golgi apparatus, have been identified (Bonifacino and Traub 2003). However, we still know little about the signals involved in yeast in anterograde trafficking from the Golgi apparatus to the plasma membrane as opposed to Golgi to vacuolar pathway trafficking. For example, we still do not know why 
transporters of the same family display sometimes different final intracellular locations. This is true of the Smf, Zrt and Ftr families of metal transporters, which include proteins resident in the plasma or vacuolar membranes, calcium P-type ATPases located in the Golgi (Pmr1p) or vacuolar (Pmc1p) membrane, and some closely related ABC transporters present in the plasma (Yor1p) or vacuolar (Ycf1p) membranes (Van Belle and André 2001). Studies of the trafficking of yeast Golgi, endosomal or vacuolar transporters are just beginning and will therefore not be covered here. However, we will cite the recent example of Ycflp (yeast cadmium factor 1), a prototype of the $\mathrm{ABC}$ transporter superfamily. This vacuolar transporter undergoes posttranslational processing by vacuolar proteases. It has an N-terminal hydrophobic extension (with five transmembrane spans) that appears to be required for vacuolar targeting (Mason and Michaelis 2002).

Although much is known about the routes and actors involved in ER insertion, ER-toGolgi, and Golgi-to-vacuole trafficking, we know little about Golgi-to-cell surface sorting. Exocytic cargos have been reported to be transported to the yeast plasma membrane by at least two routes, involving two vesicle populations with different densities, carrying different types of cargo (Harsay and Bretscher 1995; David et al. 1998). It was recently shown that at least one branch of the secretory pathway passes through endosomes before reaching the cell surface. The plasma membrane ATPase seems to be packaged directly in low-density secretory vesicles (LDSV), which bud from the Golgi apparatus, whereas the soluble glycoproteins invertase and acid phosphatase seem to be transported from the Golgi to an endosomal compartment, and then packaged in high-density secretory vesicles (HDSV) (Gurunathan et al. 2002; Harsay and Schekman 2002). Clearly, defects in the targeting of a cargo via one of these pathways may lead to its sorting via the other pathway. This situation has for instance been reported for a mutant Pma1p protein missorted from the Golgi apparatus directly to the vacuole in wild-type cells but retargeted to the plasma membrane in mutants defective in Golgi-to-endosome targeting (Arvan et al. 2002). It is unclear whether most plasma membrane transporters are packaged directly in LDSV, like Pma1p, or whether they pass through endosomes on their way to the plasma membrane. As yet, information is currently available only for the trafficking of the tryptophan permease Tat2p, which may pass through early endosomes on its way to the plasma membrane (Umebayashi and Nakano 2003). However, it is unknown whether this protein is finally recovered in LDSV or HDSV.

\section{2 Downregulation of plasma membrane transporters and channels}

\section{2. 1 Discovery of ubiquitin-dependent internalization of yeast plasma membrane transporters}

Studies on endocytosis in mammalian cells have focused primarily on receptors (Mukherjee et al. 1997), with far fewer dealing with transporters. In yeast, major insight into the mechanisms of endocytosis have been provided by studies of two G-protein-coupled receptors (GPCRs), the $\alpha$ - and a-factor receptors, Ste2p and Ste3p, respectively (Riezman 1998; D'Hondt et al. 2000), but a number of reports concerning the endocytosis of transporters have also made a major contribution to advances in our understanding of endocytosis. In the last few years, it has become clear that, like ligand-induced receptor endocytosis, the internalization of transporters is highly regulated by numerous factors, including the substrates and nutrient availability. We will first describe the actors and mechanisms generally involved in the internalization of transporters and will then deal with subsequent 
vacuolar targeting via endosomes and the mechanisms regulating internalization, focusing mainly on yeast. The discovery of the role played by ubiquitin, and the identification of the ubiquitin ligase Rsp5p as a key player in these processes in particular, is clearly one of the most important advances in our understanding of the downregulation of transporters (Rotin et al. 2000; Hicke and Dunn 2003).

Protein ubiquitylation is a posttranslational modification in which a 76-amino acid polypeptide, ubiquitin, is attached to the $\varepsilon$-amino group of lysine residues in target proteins. This process involves sequential E1 (ubiquitin-activating), E2 (ubiquitin-conjugating or UBC) and E3 (ubiquitin-protein ligase) enzyme activities, with the ligase carrying out the important task of target recognition. E3 are classified into several families. RING-finger E3s act as a scaffold, bringing together E2 and the substrate. HECT (homologous to E6AP C-terminus) domain E3s are the only class of E3 directly involved in catalysis: the activated ubiquitin is transferred from E2 to an internal Cys residue on E3 and is then conjugated to a lysine residue within the target. Proteins may be modified by monoubiquitylation: the conjugation of a single ubiquitin to one lysine or to each of several lysines in the target. Monoubiquitylation is involved in histone modification, and in several trafficking events. As ubiquitin itself carries several conserved acceptor lysines - notably Lys48 and Lys63 - multiubiquitin chains are frequently formed (Fig. 2). K48-linked multiubiquitin chains, at least four ubiquitin units long, are potent targeting signals leading to the recognition and subsequent degradation of target proteins by the $26 \mathrm{~S}$ proteasome, a large multisubunit protease complex. K63-linked ubiquitin chains are involved in other functions, including DNA repair, the activation of translation and of specific kinases, and endocytosis. Ubiquitylation is a dynamic process. Deubiquitylating enzymes (DUBs) cleave ubiquitin from proteins and disassemble ubiquitin chains. Several such enzymes are involved in endocytosis (Weissman 2001; Glickman and Ciechanover 2002).

The pheromone transporter Ste6p was the first yeast plasma membrane transporter shown to be ubiquitylated. As ubiquitylated forms of this transporter accumulate in mutants defective in endocytosis, it was suggested that ubiquitin may serve as a signal triggering the endocytosis of Ste6p (Kolling and Hollenberg 1994a). Subsequent work on two other yeast transporters fully supported this model. Early studies by Grenson and coworkers showed that ammonium triggers the rapid downregulation ("inactivation") of several yeast amino acid permeases, including the general amino acid permease Gap1p. Several npi (nitrogen permease inactivator) mutants have been isolated in which Gap1p is not inactivated by ammonium (Grenson 1992). Cloning and sequencing of the NPII gene showed it to encode the ubiquitin protein ligase Rsp5p (Hein et al. 1995), a member of the Hect family of E3s (Scheffner et al. 1995). NPI2 was found to encode Doa4p (Springael et al. 1999b), a ubiquitin isopeptidase (Papa and Hochstrasser, 1993). It was then shown that both Gap1p and another transporter, the Fur4p uracil permease, undergo Rsp5p-dependent ubiquitylation, and that this modification is required for their internalization (Galan et al. 1996; Springael and André 1998) (Fig. 3) and subsequent proteasome-independent vacuolar degradation (Galan et al. 1996). Another study carried out at about the same time showed that a ubiquitin ligase of the same family, the mammalian Nedd4, plays a critical role in the ubiquitylation and downregulation of a plasma membrane channel, ENaC (Schild et al. 1996 ; Staub et al. 1997).

Rsp5p and Nedd4 belong to a subfamily of Hect domain ligases that harbor a $\mathrm{C} 2$ domain, followed by two to four WW domains in their N-terminal regions (Rotin et al. 2000). The C2 domain is a 120-amino acid sequence that has been shown to bind phospholipids and proteins in a $\mathrm{Ca}^{++}$-dependent manner in several proteins, including Nedd4 (Plant et al. 1997; 
Plant et al. 2000). WW domains are 40-amino acid protein-protein interaction modules that bind Pro-rich ligands of various types. WW domains can be classified into four different subtypes on the basis of binding specificity. Two-hybrid and systematic phage display experiments indicated that the Rsp5p and Nedd4 WW domains are group I WW domains that bind PPxY motifs (Chang et al. 2000; Rotin et al. 2000). Rsp5p, the only member of this protein family in $S$. cerevisiae, also has functions unrelated to endocytosis. Its essential function is the ubiquitylation of two transcription factors, Spt23p and Mga2p, leading to their processing by the proteasome and subsequent nuclear import (Hoppe et al. 2000; Shcherbik et al. 2003). As Rsp5p is required for viability, some of its functions have been studied using thermosensitive mutants. However, for many studies on the endocytosis of transporters, the original npil mutant, which grows well despite having a modified promoter that decreases Rsp5p protein levels by a factor of $>10$ (Hein et al. 1995; Springael and André 1998) proved to be a very useful tool.

It soon became clear that ubiquitylation is a prerequisite for the internalization of many transporters, and for the ligand-induced and constitutive internalization of the $\alpha$ - and a-factor receptors, respectively, and that Rsp5p is the only ubiquitin protein ligase involved in this posttranslational modification (Rotin et al. 2000; Hicke and Dunn 2003; Horak 2003). Evidence that ubiquitin acts as an internalization signal is provided by the following observations (Rotin et al. 2000; Hicke and Dunn 2003; Horak 2003): 1) ubiquitin conjugates of plasma membrane proteins accumulate in mutants deficient for the internalization step of endocytosis, as reported in precursor studies on Ste6p, the a-factor transporter (Kolling and Hollenberg 1994a); 2) internalization is impaired in rsp5 / npil mutant cells, in cells deficient in E2s of the Ubc1/4/5 family that may be involved in transferring ubiquitin to Rsp5p, and after the mutation of target lysines in various transporters; 3) The N-terminal in-frame fusion of ubiquitin restores some internalization of mutant transporters (Blondel et al. 2004) and receptors (Terrell et al. 1998; Roth and Davis 2000) lacking their own usual targets Lys residues, and of a wild-type transporter in npil cells (Blondel et al. 2004) (representative example Fig. 3). Similarly, the in-frame fusion of ubiquitin increases the rate of turnover of the stable Pma1p (Shih et al. 2000). 4) The signal mediating the ubiquitylation and downregulation of one particular plasma membrane protein (Ste6p) has also been shown to be functional for another plasma membrane protein (Kolling and Losko 1997).

\section{2. 2 Ubiquitin-dependent internalization of mammalian channels}

At about the same time that it was discovered that Rsp5p was crucial for the downregulation of yeast plasma membrane proteins, it was demonstrated that Nedd4 plays a key role in downregulation of the amiloride-sensitive sodium channel, ENaC. This demonstration was the result of studies investigating the origin of a specific disease, Liddle syndrome, an inheritable severe form of hypertension caused by an increase in the cell surface activity of $\mathrm{ENaC}$. $\mathrm{ENaC}$ is essential for sodium management in the kidney. This channel consists of three subnits $(\alpha, \beta, \gamma)$, all of which have two transmembrane spans. Patients with Liddle syndrome were found to have deletion or point mutations affecting the C-terminal tails of the $\alpha$ or $\beta \mathrm{ENaC}$ subunits, in a region containing a short Pro-rich conserved motif, PPxY (PY) (Schild et al. 1996). A two-hybrid approach, with the C-terminus of the $\beta$-subunit as bait, was used to search for partners binding this motif. This approach led to identification of the WW domains of the ubiquitin protein ligase Nedd4. Consistent with this observation, $\mathrm{ENaC}$ has been shown to be regulated by ubiquitylation, primarily on a cluster of lysine residues at the $\mathrm{N}$-terminus of the $\gamma$ subnit. Mutations affecting these lysines impaired channel ubiquitylation and increased channel density at the cell surface (Staub et al. 1997). Moreover, 
the overproduction of a catalytically active form of Nedd4 in a Xenopus oocyte expression system results in the inhibition of ENaC activity, whereas the overproduction of an inactive form does not; this inhibition was found to depend on ENaC PPxY motifs (Staub et al. 1997). Thus, Nedd4 WW domains interact with ENaC via its PY motifs, leading to the ubiquitylation and subsequent downregulation and lysosomal degradation of this protein (reviewed in (Rotin et al. 2000)). Nedd4 exists as two isoforms: Nedd4-1 and Nedd4-2. Subsequent studies showed that Nedd4-2, which can be coimmunoprecipitated with $\mathrm{ENaC}$, is the isoform responsible for ENaC regulation (Kamynina and Staub 2002). It is tempting to speculate that $\mathrm{ENaC}$ downregulation results from Nedd4-2-dependent cell-surface ubiquitylation. However, this remains to be demonstrated and ubiquitylation at other cellular locations is possible given the role demonstrated for ubiquitylation at other steps of intracellular trafficking (see below).

Two other channels also appear to display ubiquitin-dependent downregulation controlled by E3s of the Nedd4 family. The cardiac voltage-gated Na+ channel (rH1), which contains PY motifs, is also negatively regulated by Nedd4 when produced in Xenopus oocytes (Abriel et al. 2000). The chloride channel ClC5 has a PY motif that is critical for its downregulation in response to interaction with an E3 of the Nedd4 family (Schwake et al. 2001). The involvement of ubiquitin ligases of the Nedd4 family in the downregulation of plasma membrane proteins is not limited to channels: some receptors are also substrates.

Although no mammalian plasma membrane transporters have yet been described to undergo ubiquitylation, or ubiquitin-dependent downregulation, posttranslational modifications of this type have been reported for two mammalian glucose transporters, GLUT1 and GLUT4. Two-hybrid systems demonstrated interaction between a conserved 11amino acid sequence in these transporters and a Ubc-like enzyme, Ubc9, responsible for conjugation with the ubiquitin-like protein, sentrin. Indeed, both transporters were found to be modified by sentrin, and were regulated in opposite ways by Ubc9. The overproduction of Ubc9 resulted in an increase in the amount of GLUT1 and a decrease in the amount of GLUT4. The authors suggested that these effects might be due to effects on intracellular pools of these transporters, rather than on plasma membrane-located transporters (Giorgino et al. 2000). Additional information is now required to determine the precise role in trafficking of this currently unique example of sentrin modification of a transporter.

\section{2. 3 Mechanisms involved in the ubiquitylation of yeast plasma membrane transporters}

We still know little about the structural motifs mediating the recognition of various substrates -including transporters- by their specific ubiquitin ligases. The interaction may be direct or mediated by accessory proteins. The recognition motif on the substrate may be part of or separate from the region carrying the target Lys (Glickman and Ciechanover 2002). Thus, the interaction between ENaC and Nedd4 appears to be a textbook example, in which the $\mathrm{C} 2$ and WW domains are involved in enzyme localization and PPxY-dependent substrate recognition, respectively, with the target Lys distinct from the recognition site, and located elsewhere in the substrate. However, this is not a general rule concerning the functioning of Nedd4. The interaction of Nedd4 with another of its substrates, the insulin-like growth factor receptor, is mediated by an adapter, Grb10, which interacts with the Nedd4 C2 domain (Vecchione et al. 2003). Nedd4 has also been reported to interact in a WW-dependent way both in vivo and in vitro, with a substrate lacking PPxY, it has also been shown to interact with PPxY-containing proteins that are unlikely to be substrates (Murillas et al. 2002). 
Although much is already known about the putative ubiquitylation signals in yeast transporters and, to a lesser extent, the role played by Rsp5p domains in this process, we still have no clear overall picture of the way in which Rsp5p interacts with transporters. We summarize below what is currently known and what remains to be determined.

\section{Factors required for ubiquitylation: Rsp5p domains and partners}

The C2 domain of Rsp5p has been shown to be required for location of the protein within the cell, in both plasma membrane invaginations and endosomes (Wang et al. 2001). Deletion of the C2 domain has been reported to impair fluid-phase endocytosis (Dunn and Hicke 2001) and to delay the internalization of both Gap1p and Fur4p, with no major effect on the ubiquitylation of these transporters (Springael et al. 1999a; Wang et al. 2001). This suggests that Rsp5p may, in addition to its enzymatic function, play a role in endocytosis. WW domains in Rsp5p interact with certain soluble substrates in a PPxY-dependent way (Wang et al. 1999). Point mutations affecting conserved residues in WW domains have shown that the WW3 domain and, to a lesser extent, the WW2 domain of Rsp5p are critical for Fur4p ubiquitylation and internalization, whereas the WW1 domain is not required [(Gajewska et al. 2001), and Marchal and Urban-Grimal, unpublished data)]. These findings suggest that the WW2 and WW3 domains of Rsp5p are involved, directly or indirectly, in Fur4p recognition. However, neither Fur4p nor any other yeast plasma membrane Rsp5p substrate has an obvious PPxY motifs that could accommodate the direct binding of Rsp5p WW domains. Thus, there may be other, currently unknown motifs involved in the interaction of Rsp5p via its WW domains, or Rsp5p may interact with its plasma membrane substrates via its other domains, or with the assistance of adaptors.

Candidate adaptors for the yeast Rsp5p protein include the Bul proteins. Bullp interacts physically with Rsp5p via its PPxY motif, and Bul2p is a homolog (Yashiroda et al. 1996; Yashiroda et al. 1998). Deletion of BUL1 and BUL2 impairs cell-surface ubiquitylation and downregulation of several transporters, Gap1p (Soetens et al. 2001) (Fig. 1), and Fui1p (C. Volland, personal communication). Thus, Bul proteins may be involved in the recognition of some Rsp5p substrates. However, it remains unclear whether these proteins are involved in the ubiquitylation of all plasma membrane Rsp5p substrates. The two Bul proteins clearly differ in their properties. The low- and high-affinity tryptophan permeases, Tat $2 p$ and Tat $1 p$, both display Rsp5p-dependent downregulation if yeast cells are subjected to high pressure. The deletion of $B U L 2$ protected Tat $2 \mathrm{p}$ against this downregulation, whereas the deletion of BUL1 did not and Tat1p downregulation was not prevented by the deletion of either gene (Abe and Iida 2003).

\section{Ubiquitylation signals and target lysines}

Whether Rsp5p interacts with transporters via the Bul proteins or via other partners, this interaction must also involve sequence motifs in the transporters. Investigations aiming to define the motifs in yeast transporters required for their ubiquitylation have revealed that an acidic stretch in the linker region connecting the two halves of the ABC-transporter Ste6p (Kolling and Losko 1997), and an N-terminal acidic PEST-like sequence in Fur4p (Marchal et al. 1998) and in the maltose permease Mal61p (Medintz et al. 2000) are essential. Many yeast transporters are phosphorylated (Table 1), and phosphorylation, notably within PEST sequences, is frequently linked to ubiquitylation (Glickman and Ciechanover 2002). This has led to investigation of the effect of transporter phosphorylation on downregulation. A point mutation affecting a potential PKC phosphorylation site in Mal61p (S295A) outside a PEST 
sequence has been shown to protect against internalization and degradation (Brondijk et al. 1998). The PEST sequence of Fur4p displays serine phosphorylation, a modification required for permease ubiquitylation at nearby lysines (Marchal et al. 1998; Marchal et al. 2000). Phosphorylation in this sequence is partly dependent on the redundant Yck1p/Yck2p casein kinase I homologs (Fig. 1) (Marchal et al. 2000). A critical threonine in the linker region of Ste6p is also important for phosphorylation, efficient ubiquitylation, and internalization (Kolling 2002). The acidity of ubiquitylation signals may therefore be affected by phosphorylation events. There is no general simple relationship between transporter phosphorylation and downregulation, and the role of the Yck kinases does not seem to be simple. The ABC transporter, Pdr5p, which has been reported to undergo in vitro phosphorylation mediated by Yck1/2, was found to be unstable in Yck1/2-deficient cells (Decottignies et al. 1999), as was the Fur4p variant lacking phosphoacceptor sites in the PEST sequence (Marchal et al. 2000). This suggests that Yck1/2 may be involved in the negative regulation of certain components of the endocytic machinery.

In addition to these sequences potentially important for recognition by the ubiquitylation machinery -Rsp5p or its partners- the critical Lys residues involved in Rsp5pdependent ubiquitylation have been identified in a number of cases (Table 1). Fur4p and Gap1p are ubiquitylated on two Lys in their N-terminal regions (Marchal et al. 2000; Soetens et al. 2001) (Fig. 1), Zrt1 is ubiquitylated on a single Lys in an intracellular loop (Gitan and Eide 2000), and in the Bap2p and Tat2p amino-acid permeases, the replacement of five and six Lys, respectively, in the $\mathrm{N}$-terminal region protects against ubiquitylation (Beck et al. 1999; Omura et al. 2001b; Soetens et al. 2001). Strikingly, in Fur4p, the Ser (Ser42, 43, 45, 55,56 ) that must be phosphorylated for subsequent Fur4p ubiquitylation (Marchal et al. 1998) lie very close to the target Lys (K38, 41) (Marchal et al. 2000), a situation similar to that described for Ste2p, in which an acidic, Ser-rich sequence, SINNDAKSS, is the target of both phosphorylation and ubiquitylation (Hicke et al. 1998). Similarly, the unique target Lys in the Zrt1p zinc transporter is 14 amino acids away from a short acidic sequence containing several serines required for Zrt1p ubiquitylation (Gitan et al. 2003).

The data described above were obtained by extensive mutagenesis (introduction of deletion and point mutations) of individual genes, for analysis of the downregulation of the corresponding proteins in defined physiological conditions known to trigger their ubiquitylation. Advances in this field were recently accelerated by the use of proteomics (Peng et al. 2003). Attempts have been made to identify all the ubiquitylated proteins in cells with 6His-tagged ubiquitin as their sole ubiquitin. Ubiquitylated conjugates were purified by retention on nickel, and analyzed by multidimensional liquid chromatography coupled with tandem mass spectrometry. In a total of 1075 potentially ubiquitylated proteins, 110 precise ubiquitylation sites were identified in 72 ubiquitin-protein conjugates. These proteins included 12 plasma membrane transporters (Table 1), with this specific group of proteins comprising a strikingly high proportion of the total. These transporters include three proteins that have already been shown to be ubiquitylated (Gap1p, Pdr5p and Ste6p) (Kolling and Hollenberg 1994a; Egner and Kuchler 1996; Springael and André 1998) and three others shown to undergo Rsp5p- and/or ubiquitin-dependent endocytosis (Hxt6p, Hxt7p, and Jen1p) (Krampe et al. 1998; Paiva et al. 2002). This bias probably reflects the higher abundance of the corresponding ubiquitin conjugates. This is consistent with the observation that the vacuolar degradation of proteins in which ubiquitylation acts as an endocytic signal is much slower than the proteasome-dependent degradation of known short-lived ubiquitylated proteins, such as cyclins, which were not recovered in this study. Strikingly, the target Lys identified in these transporters lie in very acidic motifs, often rich in Ser residues. Sixteen phosphorylation 
sites (mostly Ser, several Thr, and $2 \mathrm{Tyr}$ ) were also identified within nine transporters. Some of these sites are clustered (for instance Ser306, Thr304, Ser324 and Thr319 in Pho84p). For two of these transporters, Pho84p and Pdr5p, the ubiquitylated target Lys lie very close to the identified phosphorylated amino acids, as reported for Fur4p. Precise analysis and comparison of the sequences surrounding the target Lys may help us to understand how these motifs fit into the catalytic site of the corresponding E3, probably Rsp5p, and would undoubtedly stimulate new investigations. Data for cells cultured in standard conditions, should be complemented with data obtained in defined physiological conditions. It will be necessary to define, for instance, whether the identified target Lys are ubiquitylated at the plasma membrane, as ubiquitylation is also involved in other trafficking steps. The target Lys in Gap1p identified in this study (Lys76) differs from the two target Lys (Lys9, Lys16) shown to be ubiquitylated following the addition of ammonium to cells cultured in nitrogen-poor medium (Soetens et al. 2001), and its function remains to be determined. It may well correspond to an ubiquitylation occuring at an intracellular location. In another proteomics project based on the comparative analysis of wild-type cells and mutant cells deficient in an ERAD component, a large number of ubiquitylated proteins were identified, a number of which correspond to endogenous ERAD substrates (Hitchcock et al. 2003). About 40 ubiquitylation sites were defined including 10 in plasma membrane or vacuolar transporters (Table 1).

Although the proteomic approach requires complementary analyses, it constitutes an important step in determining whether the ubiquitylation sites in plasma membrane transporters display some signature. The data currently available highlight the link between the phosphorylation and ubiquitylation of plasma membrane transporters lacking PPxY motifs. It is tempting to think that Rsp5p may be able to recognize any of its target lysines provided that they are uncovered following a change in the conformation of the substrate transporter due to phosphorylation at a nearby site. The roles of the various domains of Rsp5p in this process, including the catalytic domain that interacts with the ubiquitylation sites, remain to be defined. Analysis of the potential interactions of plasma membrane transporters with the entire Rsp5p protein, its domains, or partners, is required.

\section{Mono- and/or polyubiquitylation of yeast plasma membrane transporters}

Yeast plasma membrane transporters are classical endocytic cargoes that are targeted for vacuolar degradation after internalization (Kolling and Hollenberg 1994a; Lai and McGraw 1994; Volland et al. 1994; Egner et al. 1995). The first studies on ubiquitindependent endocytosis indicated that despite their ubiquitylation, transporters were not recognized and degraded by the proteasome (Galan et al. 1996; Horak and Wolf 1997; Kolling and Losko 1997). This finding was accounted for in some cases by the addition to target Lys of one ubiquitin moiety, or of short chains of ubiquitin residues only two or three residues long (Fur4p, Gap1p, Mal61p, Zrt1p) as deduced from ubiquitylation patterns on western blots probed with transporter-specific antibodies (Galan and Haguenauer-Tsapis 1997; Medintz et al. 1998; Springael et al. 1999b; Gitan and Eide 2000). It was then shown that for Fur4p and Gap1p, chain extension occurred by means of a ubiquitin Lys63 bond (Galan and Haguenauer-Tsapis 1997; Springael et al. 1999b) (Fig. 2). This was demonstrated by comparing the ubiquitylation patterns of transporters in cells with wild-type ubiquitin, or ubiquitin unable to make Lys63-linked ubiquitin chains as the sole source of ubiquitin (Galan and Haguenauer-Tsapis 1997). In an alternative approach, ubiquitylation patterns were determined in npi2/doa4 cells, that have low intracellular ubiquitin level (Galan and Haguenauer-Tsapis 1997; Springael et al. 1999b; Swaminathan et al. 1999), and in which 
ubiquitin-dependent processes were rescued by expression of plasmid-encoded ubiquitin variants incompetent for the formation of Lys29-, 48- or 63-linked ubiquitin chains (Galan and Haguenauer-Tsapis 1997; Springael et al. 1999b). For Gap1p and Fur4p, it is thus clear that there are too few ubiquitin conjugates for proteasome recognition, and the ubiquitin chains present are of an inappropriate type. However, multiubiquitylated transporter species have been observed in many cases (Kolling and Hollenberg 1994a; Egner and Kuchler 1996; Horak and Wolf 1997; Beck et al. 1999), but it is unclear whether these forms result from the monoubiquitylation of several Lys or the addition of chains of ubiquitin. In studies in which ubiquitin conjugates were detected by immunoprecipitation followed by western blotting, including a recent proteomic study, high molecular weight smears were observed (Kolling and Hollenberg 1994a; Egner and Kuchler 1996; Horak and Wolf 1997; Beck et al. 1999; Peng et al. 2003). However, it is unclear whether these observations reflect extensive multiubiquitylation or the aggregation of highly hydrophobic ubiquitin conjugates. We need to determine whether Fur4p and Gap1p, two of the very few $(<10)$ known ubiquitylated substrates carrying Lys63-linked ubiquitin residues, are representative of a larger class of Rsp5p plasma membrane substrates. Recent attempts to identify yeast ubiquitylated substrates by means of proteomics have shown that Lys63 chains are more abundant than previously thought (Peng et al. 2003).

The ubiquitylation of yeast plasma membrane transporters has also been characterized to determine the type of ubiquitylation required for internalization (Fig. 2). For Fur4p and Gap1p, the generation of Lys63-linked ubiquitin chains is required for efficient endocytosis (Galan and Haguenauer-Tsapis 1997; Springael et al. 1999b). Consistent with these data, a single ubiquitin fused in-frame at the N-terminus of Fur4p triggers the internalization of a variant Fur4p lacking its own target Lys (Fig. 3), but this mutant protein is internalized at a fifth the rate for the wild-type, fully ubiquitylated permease (Blondel et al. 2004). In contrast, based on the efficient rescue of Gal2p (Horak and Wolf 2001) and Mal11p (Lucero et al. 2000) internalization in doa4A cells by the production of ubiquitin incompetent for conjugation, it was concluded that monoubiquitylation is the relevant signal for these two transporters. The reasons for this difference remain unclear. It is also unclear whether Rsp5p, which may mediate the monoubiquitylation of a membrane-bound ER substrate, Spt23p (Hoppe et al. 2000), or Lys63-linked ubiquitin modification in at least two transporters, and which is required for polyubiquitylation leading to proteasomal degradation of the soluble Rbp1p (Beaudenon et al. 1999), makes use of different adapters for these different ubiquitin modifications.

\section{2. 4 Plasma membrane-to-vacuole targeting of yeast transporters}

\section{Internalization}

Major progress in deciphering the role of ubiquitin as a sorting signal at various steps in intracellular trafficking was achieved with the discovery of ubiquitin-binding proteins that also contained variable effector domains (Katzmann et al. 2002; Bonifacino and Traub 2003). These proteins include Ede1p (the closest homolog of the mammalian Eps15), and the homologs Ent1p and Ent2p (yeast epsins). Ede1p was initially shown to be invoved in the internalization of Fur4p (Gagny et al. 2000), whereas Ent1/2 were identified as responsible for the internalization of Ste6p (Wendland et al. 1999). Ede1p has one UBA (ubiquitinassociated) motif and Ent1/2 carry two UIM (ubiquitin-interacting motif) (Shih et al. 2002). Both these domains are small (20-40 amino acids) and are known or predicted to be $\alpha$-helical. 
The Ent1/2 UIM domains have been reported to bind ubiquitin in vitro (Shih et al. 2002), and both Ent1p and Ede1p have been reported to bind membranes in a ubiquitin-dependent fashion (Aguilar et al. 2003). Several UBA and UIM domains have been reported to bind polyubiquitin chains with an affinity higher than that with which they bind to monoubiquitin. The nature of the ubiquitin chains recognized has generally not been investigated, but the UBA domain of Rad23p has been shown to bind Lys48-linked ubiquitin chains preferentially (Raasi and Pickart 2003). Thus, the possible influence of the type of ubiquitylation of membrane-bound endocytic cargoes on their potential interaction with ubiquitin-binding proteins is unknown. The Ent $1 / 2$ proteins also carry a clathrin-binding motif and an Nterminal ENTH (epsin N-terminal homology) domain. This type of domain has been shown to bind and to penetrate into membranes, causing them to curve. It has therefore been suggested that epsins act as adaptors in the selection of ubiquitylated cargos, clathrin binding, and membrane bending (Wendland 2002). This proposed role is similar to that of the main AP2 adaptors for non ubiquitylated cargos in mammals. Homologs of AP2 subunits have been identified in yeast, but play no role in internalization (Huang et al. 1999).

Despite these recent advances in the characterization of several proteins involved in the internalization step of endocytosis, and in other investigations in this field, this process remains poorly understood, for both transporters and other cargoes (recent review (D'Hondt et al. 2000)). Internalization is thought to require correct organization of the actin cytoskeleton, and many transporters accumulate as ubiquitylated conjugates in various mutants with impaired cytoskeleton organization (D'Hondt et al. 2000). There is still some debate about the function of clathrin in the internalization of yeast receptors because point mutations or deletions in the clathrin heavy chain gene $(\mathrm{CHCl})$ decrease receptor internalization by 30 $50 \%$ (reviewed in (Baggett and Wendland 2001)). Similarly, internalization is partly inhibited in at least one transporter, maltose permease (Penalver et al. 1999). The role played by clathrin in the internalization of other transporters is unknown. In contrast, a number of reports have described the clathrin-dependent internalization of plasma membrane transporters in mammals. However, little is known about the type of adaptor involved in the recognition of internalization signals in latter proteins.

\section{Trafficking to endosomes and entry into multivesicular bodies (MVB)}

Following their internalization, all endocytic ubiquitylated yeast plasma membrane cargoes are transported through several endocytic intermediates known as early (or post-Golgi endosomes, PGE), and late endosomes (or prevacuolar endosomes, PVE), where they meet membrane proteins from the Golgi apparatus, and are then delivered to the vacuole lumen for degradation. One of the first post-internalization events appears to be segregation into specific lipid domains distinct from plasma membrane lipid domains. For example, Fur4p, which is primarily present in rafts when at the plasma membrane, whether ubiquitylated or not, is mostly recovered in non-raft domains once it has entered endosomes (Dupre and HaguenauerTsapis 2003).

Many of the factors involved in late steps of endosome-to-vacuole trafficking have been identified by genetic screens based on the missorting in the medium of carboxypeptidase Y (CPY), a vacuolar protease. In some cases, screens based on several transporters were used. Some thermosensitive mutations affecting the essential plasma membrane ATPase Pma1p, or the arginine permease Can $1 \mathrm{p}$, result in the direct delivery of these proteins, at $37^{\circ} \mathrm{C}$, from the Golgi complex to endosomes/vacuoles for degradation. Genetic selection for suppressors restoring normal growth at $37^{\circ} \mathrm{C}$, or the reacquisition of normal sensitivity at $37^{\circ} \mathrm{C}$ to 
canavanin, a toxic analog of arginine, led to the identification of known or new vps (vacuolar protein sorting) mutants (Luo and Chang 1997; Li et al. 1999). For example, SPT22/VPS23, encoding a protein conserved from yeast to man carrying a Ubc-like domain, which plays a crucial role in the generation of and sorting to multivesicular bodies (MVBs) was first identified by screening for the reacquisition of normal sensitivity at $37^{\circ} \mathrm{C}$ to canavanin ( $\mathrm{Li}$ et al. 1999).

Understanding the molecular mechanisms involved in the formation of and sorting to MVBs has been a major goal in the last three years (Piper and Luzio 2001; Katzmann et al. 2002). MVBs form in late endosomes, when the limiting membrane invaginates and buds into the lumen of the organelle to form internal vesicles (Fig. 1). During this process, a subset of the membrane proteins within the limiting membrane of the endosome is sorted to these invaginating vesicles. Subsequent fusion of the mature MVB with the lysosome/vacuole results in the delivery of the internal vesicles and their associated cargoes to the lumen of the lysosome/vacuole, where they are degraded by vacuolar proteases and lipases. Proteins that remain in the limiting membrane of the MVBs are delivered to the limiting membrane of the lysosome/vacuole. This process thereby separates proteins destined for the lumen of the lysosome/vacuole from proteins destined for the limiting membrane of this organelle. MVB formation is impaired in a subset of vps mutants, the vps class $\mathrm{E}$ mutants, which accumulate an exaggerated late endosome known as the class $\mathrm{E}$ compartment, in which endocytic and membrane-bound Golgi proteins remain trapped. Class E Vps proteins, conserved from yeast to man, have been shown to be involved in both the formation of MVBs and sorting to MVBs.

Endocytic cargoes, including plasma membrane transporters, are sorted to the internal vesicles of MVBs (Fig. 1). They are therefore directed to the vacuolar lumen (representative example Fig. 3), together with a subset of biosynthetic cargoes, such as carboxypeptidase S (Cps1p), a transmembrane protein that is processed to generate its active enzymatic form in the vacuolar lumen. Various studies have demonstrated that the ubiquitylation of MVB cargoes plays a critical role in sorting to the internal vesicles of MVBs. Early data concerning the fate of plasma membrane or vacuolar transporters significantly contributed to this demonstration. Ste6p was shown to accumulate at the vacuolar membrane rather than being released into the vacuolar lumen in doa4 cells, which have low levels of ubiquitin, and to be correctly sorted if ubiquitin was overproduced (Losko et al. 2001). Fth1p, a vacuolar iron transporter with multiple transmembrane spans is normally delivered to the vacuolar membrane but was shown to be sorted to the vacuolar lumen following the in-frame fusion of ubiquitin (Urbanowski and Piper 2001). Cps1p, which has a single transmembrane span, primarily undergoes monoubiquitylation and this modification is sufficient for correct sorting to MVBs (Katzmann et al. 2001). Little is known about the type of ubiquitylation required for the sorting to MVBs of transporters with multiple transmembrane spans originating from the plasma membrane. However, the ubiquitylation status of the protein acquired at the plasma membrane clearly leads to efficient MVB sorting. In-frame ubiquitin has been shown to restore the plasma membrane internalization of Fur4p lacking its normal target Lys to some extent. Nonetheless, some of this chimeric protein was retained at the vacuolar membrane, suggesting that monoubiquitylation is not sufficient for the efficient MVB sorting of this class of proteins (Fig. 3) (Blondel et al. 2004).

Ubiquitylated cargoes arriving from the plasma membrane and the Golgi apparatus are thought to interact successively with several class E proteins. The first are two Vps class E proteins carrying UIM domains, Hse1p and Vps27p, which form a complex (Bilodeau et al. 2002). Cargoes are then recognized successively by other class $E$ proteins that form three 
high-molecular weight complexes, referred to as ESCRT I, II and III (endosomal sorting complexes required for transport I, II and III) (Katzmann et al. 2002). Vps23p, which has been shown to interact with Vps27p (Bilodeau et al. 2003), is the only component of the ESCRT complexes with a ubiquitin-binding region, the Ubc-like domain (Katzmann et al. 2002). Most of the proteins comprising these complexes were identified by the cloning of identified class E vps gene, systematic purification in the native state of the various corresponding proteins, and analysis of coimmunoprecipitated partners.

One of the proteins identified in specific screens for Vps class E proteins, Vps31p/Bro1p, which is associated with the ESCRT III complex (Odorizzi et al. 2003), was also isolated in two genetic screens based on amino-acid uptake. Mutations in BROI have been identified in many suppressors of phenotypes associated with a lack of the amino acid sensor Ssylp (Forsberg et al. 2001). Curiously, mutations in BROl were also identified long ago as an $n p i$ mutant, npi3, impaired in the $\mathrm{NH}_{4}^{+}$-induced downregulation of cell-surface Gap1p. $\mathrm{NH}^{+}{ }^{+}$ induced ubiquitylation and downregulation of Gap1p was strongly inhibited in npi3 cells (Springael et al. 2002). Although these observations initially suggested a possible role for Brolp at both the plasma and late endosome membranes, further analysis of the npi3/brol mutant revealed that Gap1p was ubiquitylated and internalized by endocytosis but that its sorting to MVB was defective. A fraction of the internalized permease is thus missorted to the limiting membrane of the vacuole. However, most of the internalized Gap1p is recycled back to the plasma membrane, explaining why Gap1p remains largely active in this mutant after the addition of $\mathrm{NH}_{4}^{+}$. Interestingly, this recycling seems to be accompanied by de-ubiquitylation of the permease (Nikko et al. 2003).

Although most Vps class E proteins appear to be involved in the sorting of all MVB cargoes, the possibility of some specificity cannot be ruled out, as shown recently by the identification of new class E vps genes by means of a strategy based on the fate of the Ste6p transporter. A colony assay was developed to identify genes involved in late steps of Ste6p endocytosis. This assay was used to search for genes that, when overexpressed, caused impaired degradation of a Ste6-lacZ fusion protein. A number of known class E vps genes were recovered, and MOS10 (more of Ste6), a gene of previously unknown function was also identified (Kranz et al. 2001). Mutations in MOS10 were also recovered as suppressors of ssyl $\Delta$ phenotypes (Forsberg et al. 2001). The overexpression and deletion of MOS10 both gave a class E phenotype. MOS10 encodes a coiled-coil protein, homologous to two proteins of the ESCRT III complex, Vps20p and Snf7p. These three proteins, which form a small family, display subtle differences in function. The phenotypes of the deletion strains are not identical. For instance, snf7 $\Delta$ cells display impaired glucose signaling, whereas vps $20 \Delta$ and snf7 $\Delta$ cells display heat sensitivity for growth. These phenotypes are not displayed by mos $10 \Delta$ cells, but mos10 / mos $10 \Delta$ cells have specifically impaired filament maturation during pseudohyphal growth (Kohler 2003). Although the precise reasons for these differences in phenotype are unknown, these observations suggest that these three proteins may well preferentially affect the sorting of specific MVB cargoes.

The fate of the attached ubiquitin during the final step of MVB sorting has also been analyzed. Genetic data suggested that the Doa4p ubiquitin isopeptidase may be involved in the trafficking of endocytic cargoes. Intracellular ubiquitin concentrations are low in doa4 4 cells (Springael et al. 1999b; Swaminathan et al. 1999), and this phenotype is suppressed in mutants defective for the internalization step of endocytosis (Swaminathan et al. 1999). The potential role of Doa4p in the deubiquitylation of plasma membrane proteins was tested by 
following the fate of Fur4p-ubiquitin conjugates. Fur4p accumulated entirely in the form of plasma membrane ubiquitin conjugates over several hours in cells in which the internalization step of endocytosis was impaired, but was recovered in deubiquitylated form in vacuoles of pep $4 \Delta$ cells, deficient in vacuolar proteases activities. In contrast, pep $4 \Delta$ doa $4 \Delta$ cells accumulated vacuolar Fur4p exclusively in the ubiquitin-conjugated form. Thus, Ub-Fur4p undergoes Doa4p-dependent deubiquitylation during plasma membrane-to-vacuole trafficking (Fig. 1). A lack of deubiquitylation did not prevent the MVB sorting of Fur4p (Dupré and Haguenauer-Tsapis 2001). Doa4p, which has been shown to accumulate in endosomes in class E vps mutants (Amerik et al. 2000), has also been shown to be involved in deubiquitylation of MVB cargoes from the Golgi apparatus (Katzmann et al. 2001). This protein is probably the central ubiquitin isopeptidase acting after the ESCRT III complex, just before the sorting of cargoes to MVBs. The delivery to the vacuole of ubiquitin-conjugated endocytic and biosynthetic cargoes in doa4d cells leads to the massive proteolytic degradation of ubiquitin, resulting in defective ubiquitin homeostasis in these cells.

In addition to the role played by ubiquitin as a sorting signal in early and late steps in the endocytosis of plasma membrane transporters, at least one additional signal has been shown to be involved in the endocytic pathway of a yeast transporter. The normal $\mathrm{NH}_{4}^{+-}$ induced downregulation of Gap1p is also dependent on a di-leucine motif present in a predicted coiled domain of the cytosolic C-terminal tail of the permease (Hein and André 1997). Interestingly, the sequence context of this di-leucine fits the structural requirements for recognition by the VHS domain of Gga proteins, recently identified as adaptors (Bonifacino and Traub 2003). Substitution mutations affecting the di-leucine or neighbouring acidic residues do not prevent $\mathrm{NH}_{4}^{+}$-induced Gap1p ubiquitylation but do impair the subsequent endocytosis and degradation of Gap1p (Springael and André 1998). It remains to be determined whether the di-leucine is required for early and/or late steps of internalization of the Gap1p permease. A role for the di-leucine late in endocytosis would suggest that Gap1p is recycled back to the cell surface if the di-leucine motif is not accessible to some VHScontaining proteins.

\section{3 Regulation of transporters and channels at membrane trafficking levels: signals and mechanisms}

We summarize below the diverse physiological situations reported to induce adaptive changes in the membrane sorting of specific transporters. We will begin by considering the numerous cases of accelerated endocytosis of a transporter in response to environmental stimuli. We will then describe situations involving controlled sorting of transporters present in the Golgi and/or endosomal membranes. We will then comment briefly on similar control systems for mammalian transporters. Finally, we will discuss models of the integration of upstream signals for control of the global trafficking of transporters.

\section{3. 1 Physiological control of the rate of internalization of transporters}

Changes in environmental conditions often lead to drastic increases in the rates of internalization of specific transporters by endocytosis, and to the targeting of these transporters to the endosome/vacuolar pathway for degradation. Furthermore, the downregulation of transporters is often accompanied by the induction of other transport systems more appropriate for the new conditions. This dynamic control of transporters has been studied in detail in both yeast and mammalian cells. 
Yeast cells possess about twenty different amino acid permeases, the substrate specificities of which overlap in many cases. It is now established that these proteins are not simultaneously active in the cells. Instead, most are differentially regulated according to the nitrogen and/or amino acid content of the growth medium. For instance, the general amino acid permease (Gap1p) (Grenson et al. 1970; Jauniaux and Grenson 1990) is the most active amino acid permease in conditions of limiting nitrogen supply, such as low levels of $\mathrm{NH}_{4}^{+}$or urea being the sole available sources of nitrogen. Gap1p is also highly active if cells are grown on proline, another poor nitrogen source. Under these conditions, the GAPl gene is strongly transcribed (Jauniaux and Grenson 1990) and the newly synthesized Gap1p is targeted to the plasma membrane, where it is active and highly stable (Grenson 1983a; De Craene et al. 2001). If a preferential source of nitrogen, such as $\mathrm{NH}_{4}^{+}$, is added at a sufficiently high concentration, the GAPl gene is repressed and pre-synthesized Gap1p is progressively removed from the cell surface by endocytosis and sorting to the MVB/vacuolar degradation pathway (Grenson 1983a; Hein et al. 1995; Springael and André 1998; De Craene et al. 2001; Nikko et al. 2003). This downregulation may also be triggered by the addition of high concentrations of amino acids (Stanbrough and Magasanik 1995). These changes of nitrogen supply conditions are thought to inactivate Npr1p, a protein kinase playing a pivotal role in the control of trafficking of several permeases including Gap1p and which is specifically active when cells grow under poor nitrogen supply conditions (Grenson, 1983b; Vandebol et al., 1987, 1990; Schmidt et al. 1998). Inactivation of Npr1p by shifting an $n p r I^{t^{s}}$ mutant to the non-permissive temperature also leads to downregulation of Gap1p (Grenson 1983b; De Craene et al. 2001). As discussed below, inactivation of Npr1p in response to good nitrogen supply conditions is dependent on the TOR signaling pathway (Schmidt et al. 1998). The Rsp5p- and Bul-dependent ubiquitylation of Gap1p on its two most N-terminal lysine residues (at positions 9 and 16) is essential for the downregulation of this protein (Hein et al. 1995; Springael and André 1998; Soetens et al. 2001) (Fig. 1). Under conditions of poor nitrogen supply, only a small fraction of Gap1p is monoubiquitylated. The addition of $\mathrm{NH}_{4}^{+}$ concomitantly increases the monoubiquitylation and polyubiquitylation of Gap1p via Lys63linked chains (Springael and André 1998; Springael et al. 1999b).

During the downregulation of Gap1p following the addition of high concentrations of amino acids, Gap1p degradation seems to be preceded by dephosphorylation (Stanbrough and Magasanik 1995). In contrast to Gap1p, other broad-specificity amino-acid permeases such as Agp1p, Tat2p, Tat1p and Bap2p are induced, with increases in transcription of the corresponding genes in response to the addition of amino acids (Iraqui et al. 1999). These permeases therefore take on the functions of Gap1p. This induction is dependent on the Ssy1p permease-like sensor of external amino acids (Boles \& André, 2004, this issue). Unlike Gap1p, these permeases are synthesized and active in cells grown on YPD medium. However, Bap2p and Tat $2 p$ are not exclusively located at the cell surface under these conditions. Instead, a significant proportion of these permeases are present in internal membranes, probably those of endosomes (Beck et al. 1999; Omura et al. 2001a; Umebayashi and Nakano 2003). The presence of large amounts of these permeases within cells may be due to their direct sorting for degradation because their amino acid substrates are present at high concentrations in the medium.

Shifting cells from YPD medium to nitrogen starvation conditions results in the derepression of Gap1p and the rapid downregulation of Tat2p and Bap2p. This 
downregulation process may also be induced by adding rapamycin, or possibly other compounds such as anesthetics (Palmer et al. 2002) or the FTY720 immunosuppressive agent (Welsch et al. 2003), to the medium. These compounds interfere with the TOR signaling pathway, which is active in favourable nutrient supply conditions (Crespo and Hall 2002). The requirements for ubiquitin, Rsp5p, and lysines in the downregulation of Tat $2 p$ and Bap2p have been extensively studied (Beck et al. 1999; Omura et al. 2001b; Umebayashi and Nakano 2003). Lysines in the $\mathrm{N}$-terminus of Bap2p, and the $\mathrm{C}$-terminal tail of this protein have been shown to play a key role in determining its fate in the endocytic pathway (Omura et al. 2001a).

The Gap1p, Bap2p and Tat2p permeases all belong to the AAP/YAT family of closely related amino acid permeases. So, what are the structural features in these permeases reponsible for determining their pattern of regulation according to nitrogen and amino acid availability? Although the C-terminal tail of Gap1p plays an essential role in $\mathrm{NH}_{4}{ }^{+}$-induced downregulation, it is not sufficient to confer a similar pattern of regulation on the $\mathrm{NH}_{4}^{+}$insensitive Can1p permease (Hein and André 1997). However, replacement of the $\mathrm{N}$-terminal tail of Gap1p with that of Bap2p confers partial sensitivity to rapamycin-induced degradation. This suggests that the $\mathrm{N}$-terminal tail, which is subject to ubiquitylation, contains sufficient information to confer nitrogen-dependent control of endocytosis (Omura et al. 2001b).

\section{Regulated endocytosis of sugar and monocarboxylate transporters}

Although glucose at high concentration is the preferred carbon source, yeast cells can also use alternative sugars such as galactose or maltose, or monocarboxylates such as lactate or pyruvate. The permeases involved in the uptake of these compounds (Gal2p, Mal61p, Jen1p) are typically induced by their own substrates and are subject to glucose-mediated inhibition (Horak 2003). If high concentrations of glucose are added to cells grown under inducing conditions, the genes encoding the induced permeases are repressed and the permeases already present at the cell surface undergo ubiquitin-dependent downregulation (Horak and Wolf 1997; Lucero and Lagunas 1997; Medintz et al. 2000; Paiva et al. 2002). The same fate has been observed for two high-affinity glucose transporters (Hxt6p, -7) and the high- to intermediate-affinity transporter Hxt2p, which are specifically induced in response to low extracellular glucose concentrations (Kruckeberg et al. 1999; Krampe and Boles 2002). For unknown reasons, the glucose-triggered downregulation of sugar permeases is more pronounced if cells are simultaneously starved of nitrogen (Lucero et al. 2002). It was recently suggested that this is due to a contribution, in this induced degradation, of the autophagy pathway (Krampe and Boles 2002).

\section{Regulated endocytosis of nucleobase transporters}

The high-affinity uracil permease (Fur4p) undergoes ubiquitylation and subsequent internalization and vacuolar degradation at basal rates in exponentially growing cells (Volland et al. 1994; Galan et al. 1996). As a result, the permease can be visualized both at the plasma membrane, and in endosomes, en route for vacuolar degradation (Dupré and HaguenauerTsapis 2001). Exposing cells to various adverse conditions - nitrogen, carbon, or phosphate starvation, inhibition of protein synthesis by cycloheximide - and the lead-up to the stationary phase of growth result in increases in the rates of ubiquitylation, endocytosis and degradation (Volland et al. 1994; Galan et al. 1996). The addition of high concentrations of uracil to the medium also decreases uracil permease levels, partly by destabilizing FUR4 
mRNA (Séron et al. 1999). This type of regulation therefore differs from the more usual transcriptional regulation displayed in the case of other permeases. Uracil also triggers rapid degradation of the existing permease by accelerating ubiquitylation and endocytosis (Séron et al. 1999) (Fig. 3). As the adverse conditions that accelerate permease turnover also lead to ribosome degradation (Warner 1999), they are likely to trigger an increase in the internal pool of uracil of catabolic origin, which may be the main signal for Fur4p downregulation.

Interestingly, the uracil-induced control of Fur4p trafficking requires direct binding of uracil to the permease, as a mutant permease with an abnormally low affinity for uracil displays impaired transport activity (Urban-Grimal et al. 1995) and is not internalized following the addition of uracil to the medium (Séron et al. 1999). These observations suggest that the recognition by transporters of their own substrates and the susceptibility of these proteins to be ubiquitylated may be intimately linked.

\section{Regulated endocytosis of metal transporters}

Metal transporters constitute another class of tightly regulated transport systems. This tight regulation is accounted for by the need for metals, at particular concentrations, and their potential toxicity at high concentration. The maintenance of correct metal homeostasis is therefore critical.

$\mathrm{Mg}^{2+}$, essential for the activation of hundreds of enzymes, is the most abundant divalent cation in cells (concentrations in the millimolar range). Total intracellular $\mathrm{Mg}^{2+}$ concentrations are tightly controlled, varying by a factor of no more than four, whereas external concentrations may change by four orders of magnitude. The yeast permease Alr1p was the first candidate $\mathrm{Mg}^{2+}$ transporter to be identified in eukaryotic cells. Alr1p is essential for the growth of yeast cells in all media except those with high $\mathrm{Mg}^{2+}$ concentrations. The exposure of cells to even standard $\mathrm{Mg}^{2+}$ concentrations leads to a dramatic decrease in the stability of this protein, which undergoes ubiquitin-dependent rapid internalization (Graschopf et al. 2001).

A similar situation has been described for $Z R T 1$, which encodes the high-affinity zinc transporter Zrt1p. ZRT1 is repressed in cells replete with zinc, and spectacular Rsp5pdependent ubiquitylation of the transporter is triggered within minutes of exposure of cells to high levels of zinc (Gitan et al. 1998; Gitan and Eide 2000).

The highly conserved Smf1p and Smf2p proteins display broad specificity for both essential and nonessential metals (manganese, cadmium, copper, iron etc.). Smf1p is rapidly internalized in the presence of manganese (Liu and Culotta 1999b).

Finally, copper is yet another essential metal required for the activity of various enzymes involved in critical areas of metabolism. Copper is also potentially toxic if it accumulates beyond cellular needs, and intracellular levels of this nutrient must therefore be precisely regulated. Copper is transported in yeast and mammals by homologous Ctr1 proteins with three predicted membrane-spanning regions that form oligomeric complexes. The yeast Ctr1p is highly responsive to copper availability, being upregulated by the Mac1p transcription factor if extracellular copper levels are low. In addition to this transcriptional regulation, the levels of Ctr1p protein at the plasma membrane are posttranslationally regulated by copper availability. Two different mechanisms have been described. At high copper concentrations (in the range of $10 \mu \mathrm{M}$ ), Ctr1p is degraded in situ at the plasma membrane, via a mechanism independent of endocytosis - an unusual situation for a plasma membrane protein. At low micromolar levels of copper, Ctr1p undergoes accelerated endocytosis (Ooi et al. 1996), a process dependent on the same transcription factor as CTRI 
induction (Yonkovich et al. 2002). For both Smf1p and Ctr1p, the potential role of ubiquitylation events in these processes has yet to be determined.

\section{Regulated endocytosis of other yeast transporters}

One of the first illustrated cases of regulation of a transporter by its own substrates was provided in the case of Itr1p, an inositol permease. Upon addition of excess inositol, the ITRI gene is repressed and pre-synthesized Itr1p undergoes accelerated endocytosis and degradation (Lai et al. 1995). Substrate-induced endocytosis has also been reported for the spermidine transporter (Kaouass et al. 1998). The high-affinity phosphate transporter, Pho84p, is also regulated by its own substrate. In the presence of low external phosphate concentrations, the PHO84 gene and other $\mathrm{PHO}$ genes involved in phosphate metabolism are derepressed, and Pho84p is targeted to the plasma membrane. If phosphate is added at high concentrations, the PHO84 gene is repressed and accelerated Pho84p endocytosis is observed (Martinez et al. 1998; Petersson et al. 1999).

\section{Regulated endocytosis of mammalian transporters}

A number of mammalian plasma membrane transporters and channels undergo regulated trafficking, involving regulated internalization in particular, as discussed in several reviews (Lee 2000; Royle and Murrell-Lagnado 2003). We therefore provide here only significant examples of different types of regulation, some of which are similar to that described for yeast plasma membrane transporters.

Substrate-regulated internalization is a common feature of mammalian metal transporters. The human copper transporter, hCtr1, which undergoes copper-stimulated endocytosis and degradation like its yeast conterpart (Petris et al. 2003) has been studied in detail. Interest in yeast and human Ctr1 recently increased with the demonstration that these transporters mediate uptake of the anticancer drug, cysplatin, which also accelerates endocytosis (Ishida et al. 2002).

The endocytosis of neurotransmitter transporters is highly regulated and enables the neuron to fine-tune transport capacity to match demands. The cocaine- and amphetaminesensitive dopamine (DA) transporter (DAT), a presynaptic plasma membrane protein responsible for the regulation of extracellular DA concentrations, undergoes endocytosis in response to protein kinase $\mathrm{C}$ (PKC) activation. Activated PKC then catalyzes DAT phosphorylation. Several transporters belonging to the monoamine branch of the same family of $\mathrm{Na}^{+} / \mathrm{Cl}^{-}$-dependent plasma membrane transporters - the serotonin (SERT), norepinephrine (NET), and epinephrine transporters - display similar PKC-dependent downregulation (Buckley et al. 2000). For a number of these transporters, PKC-dependent phosphorylation is triggered by external substrates. DAT substrates, including amphetamine, induce DAT internalization (Saunders et al. 2000). This effect of substrates is the opposite of that described for SERT (Ramamoorthy and Blakely 1999), suggesting markedly different mechanisms of regulation for two closely related transporters. Another related transporter, the $\gamma$-aminobutyric acid transporter (GAT1), undergoes substrate-induced tyrosine phosphorylation, reducing the rate of GAT1 internalization (Whitworth and Quick 2001). It is unclear how phosphorylation by different kinds of kinases can regulate the rates of internalization of target transporters in opposite ways. Similarly, although substrate-induced

regulation of the rate of internalization appears to be a common property of yeast and mammalian plasma membrane transporters, it is unknown whether the underlying 
mechanisms are similar and whether ubiquitin is important the downregulation of mammalian transporters.

Some transporters are downregulated by hormones. Extensive studies of the insulinregulated trafficking of the Glut glucose-transporters have been carried out (Hasani, this issue). In addition, $\mathrm{ENaC}$ remains a rare example of a plasma membrane protein involved in transport for which hormonal downregulation is mediated by a ubiquitylation event. ENaC activity is known to be regulated by a number of hormones, including aldosterone. This regulation seems to involve Sgk1 kinase (serum and glucocorticoid regulated kinase), a member of the Akt family of Ser/Thr kinases, which is induced by aldosterone and stimulates $\mathrm{ENaC}$. It was recently shown that Sgk1 acts directly on the Nedd4-2/ENaC interaction. Based on the observation that Sgk1 has a PPxY motif, and that Nedd4-2 includes two consensus sites for phosphorylation by Sgk1, Staub and coworkers showed that Sgk1 phosphorylates Nedd4-2 in a PPxY-dependent manner in Xenopus oocytes, and that this phosphorylation reduces the interaction between Nedd4-2 and ENaC, leading to high levels of ENaC at the cell surface, possibly as a result of impaired internalization (Debonneville et al. 2001). It is unknown whether similar mechanisms control the interaction of yeast Rsp5p with any of its plasma membrane substrates. However, such regulation appears unlikely, given the increasing number of known substrates of this single member of the Nedd4 protein family at various subcellular locations in yeast.

\section{3. 2 Physiological control of the sorting and recycling of newly synthesized transporters}

The fate of newly synthesized transporters present in the secretory pathway may also depend on environmental conditions: the protein may be targeted to the cell surface, or directly diverted to the MVB/vacuole for degradation without passing through the plasma membrane. The membrane compartment in which this control is achieved is probably the late Golgi compartment and/or a post-Golgi endosome, although additional controls in the late endosome compartment cannot be ruled out. Ubiquitin and lipid rafts appear to play crucial roles in regulating transporter sorting. On the other hand, a few yeast transporters present in internal membranes have been reported to be recycled back to the plasma membrane in response to environmental stimuli. However, in these situations, it is important to distinguish between the targeting to the cell surface of newly synthesized transporters and the recycling of internalized transporters previously present at the plasma membrane.

\section{Regulated sorting of amino acid permeases}

The first evidence for control at the sorting level of a newly synthesized yeast transporter was reported for the Gap1p permease (Roberg et al. 1997a; Roberg et al. 1997b). In cells grown on glutamate as the sole nitrogen source, Gap1p is synthesized but inactive and is located principally in the internal membranes. Most of the Gap1p synthesized in these cells was diverted from the secretory pathway to the vacuole without passing through the plasma membrane (Fig. 4). If the cells are transferred back onto urea, Gap1p activity increases slightly, even if cycloheximide is added to the medium (Roberg et al. 1997b). This increase in Gap1p activity may be due to Gap1p proteins present in the secretory pathway and that are targeted to the cell surface instead of being sorted to the vacuolar degradation pathway. Alternatively, as suggested by the authors, this increase may be due to Gap1p proteins stored in internal membranes and recycled back to the plasma membrane once the cells are transferred to conditions of poor nitrogen supply. In a subsequent study, Helliwell et al. 
(Helliwell et al. 2001) identified Bul1p and Bul2p as redundant proteins required for the direct sorting of Gap1p to the vacuole. These proteins were also found to be specifically required for the poly-ubiquitylation of Gap1p, which suggests that poly-ubiquitylation is essential for direct sorting of the permease to the vacuole, monoubiquitylation being insufficient. In the bull bul2 and rsp5 mutants, newly synthesized Gap1p normally directed to the vacuole is retargeted to the cell surface (Fig. 4).

Gap1p permease produced artificially in cells growing in the presence of high concentrations of $\mathrm{NH}_{4}{ }^{+}$is also directly sorted to the $\mathrm{MVB} /$ vacuole without passing through the cell surface. Ubiquitylation of Gap1p on lysines 9 and 16 is required for this direct sorting to the vacuole, as are the Rsp5p ubiquitin ligase and Bul proteins. Mutations altering any of these components target the Gap1p permease to the cell surface, regardless of the quality of the nitrogen source (Soetens et al. 2001). A similar phenotype has been observed in doa4 cells, which have a much smaller than normal internal ubiquitin pool, and this phenotype was suppressed by ubiquitin overproduction. Furthermore, a similar suppression was observed following the overproduction of a ubiquitin variant unable to form poly-ubiquitin chains $\left(\mathrm{Ub}^{\mathrm{RRR}}\right)$. Thus, at least under certain conditions, the mono-ubiquitylation of Gap1p seems to be sufficient for direct sorting of the permease to the vacuole (Soetens et al. 2001). Further investigations are clearly required to elucidate the actual roles of the Bul proteins and of mono- vs. polyubiquitylation in the regulated sorting of Gap1p.

It also remains to be determined whether ubiquitylation of the newly synthesized permease indeed takes place in the secretory pathway and serves as a signal for the nitrogeninduced diversion of the permease to the MVB/vacuolar degradation pathway (Helliwell et al. 2001). Alternatively, in cells growing on glutamate or high concentrations of $\mathrm{NH}_{4}^{+}$, newly synthesized Gap1p may be missorted to the late endosome without ubiquitin involvement. The permease would then undergo ubiquitylation, leading to its sorting to the MVB/vacuolar degradation pathway. If not ubiquitylated, the protein would instead be retargeted to the cell surface.

The sorting of Tat2p is also regulated by cellular nitrogen status (Beck et al. 1999). If cells growing on rich medium are shifted to nitrogen starvation conditions or if rapamycin is added to the medium, Tat $2 \mathrm{p}$ proteins present in the secretory pathway are preferentially sorted to the vacuole for degradation. Mutations impairing Tat2p ubiquitylation (npil/rsp5, npi2/doa4, replacement of five lysine residues in the N-terminal tail of the permease) or preventing access of the permease to the late endosome (pep12) or its sorting in the MVB pathway (vps27) protect it against degradation. It was recently shown that tryptophan availability in the medium plays an important role in controlling the fate of newly synthesized Tat2p (Umebayashi and Nakano 2003). In the presence of low concentrations of tryptophan, Tat $2 p$ is targeted to the cell surface, and this process is thought to involve transit of the permease through a post-Golgi or early endosome compartment. On media containing high concentrations of tryptophan, Tat $2 p$ is instead sorted from this compartment to the vacuole. Lipid rafts and ubiquitin play key roles in this regulated permease sorting. A defect in the ERG6 gene encoding S-adenosylmethionine-delta-24-sterol c-methyltransferase, an enzyme involved in a late step of ergosterol biosynthesis, leads to vacuolar sorting of the permease even in the presence of low concentrations of tryptophan. However, if access to the late endosome is further restricted by means of a pep 12 mutation, Tat $2 p$ is retargeted to the cell surface. Thus, the association of Tat $2 p$ with rafts is required for the normal targeting of this protein to the cell surface. Tat $2 p$ may be unable to associate with rafts if tryptophan is abundant in the cell, leading to its missorting to the MVB/vacuolar degradation pathway. Vacuolar sorting of Tat $2 p$ in the presence of high concentrations of tryptophan or in erg6 
mutants grown in the presence of low concentrations of tryptophan is defective in the bull mutant, in which the ubiquitylation (probably polyubiquitylation) of Tat $2 p$ is defective. Ubiquitylation seems to occur after Tat $2 p$ has left the Golgi apparatus, but it is unknown whether it occurs in the post-Golgi endosome or only once Tat $2 p$ has reached the late endosome membrane.

\section{Regulated sorting of nucleobase transporters}

Similar nutrient conditions trigger both the onset or acceleration of amino acid permease endocytosis and the direct vacuolar routing of newly synthesized proteins. A similar dual regulation system has been reported for nucleobase permeases. Uracil and uridine permeases, both of which undergo substrate-induced plasma membrane internalization, display direct vacuolar routing if synthesized de novo in the presence of their respective substrates (Séron et al. 1999; Blondel et al. 2004). The molecular mechanisms underlying this process have been studied in more detail for uracil permease. Most of the experiments were carried out with GFP-tagged wild-type or variant versions of Fur4p.

A mutant version of Fur4p with a very low affinity for uracil could not be diverted to the vacuolar pathway when synthesized de novo in the presence of uracil. Instead, the permease was targeted to the cell surface. These experiments were conducted on cells producing a GFP-tagged version of the mutant permease, together with a wild-type untagged permease to permit uracil uptake. In contrast to the fate of this mutant permease, in cases of defective ubiquitylation, and in rsp5/npil cells in particular, Fur4p synthesized de novo in the presence of uracil was able to leave the Golgi apparatus and to reach the vacuole, but was missorted to the vacuolar membrane (Fig. 3). Correct luminal delivery was restored by the inframe N-terminal fusion of ubiquitin, which also resulted in the partial diversion of Fur4p to the vacuolar pathway (Blondel et al. 2004). These data suggest that the binding of intracellular uracil to the permease promotes a conformational change preventing transport of the permease to the plasma membrane. Missorted permease then undergoes ubiquitylation, leading to its delivery to the vacuolar lumen (Blondel et al. 2004). It should be noted that defective ubiquitylation (npil cells) does not seem to prevent Fur4p from leaving the Golgi apparatus and reaching the late endosome. It is the subsequent MVB sorting of the permease that is specifically impaired. Note that all the available data are consistent with a similar model for the control by amino acids of the Gap1p permease, except that this protein is mainly re-targeted to the cell surface if not correctly ubiquitylated.

The requirements for Fur4p ubiquitylation at the plasma membrane and during Golgito-MVB sorting have been compared. Both events are Rsp5p-dependent. The ubiquitylation required for MVB sorting did not require phosphorylation of the PEST sequence of the permease (Blondel et al. 2004), whereas plasma membrane ubiquitylation did (Marchal et al. 1998). Fur4p Lys38 and Lys41, the only target Lys for plasma membrane ubiquitylation (Marchal et al. 2000) were also sites of ubiquitylation for MVB sorting, together with other Lys (Blondel et al. 2004). The type of ubiquitylation occuring during Fur4p Golgi-to-vacuole targeting has not yet been determined. Experiments conducted with an in-frame ubiquitin showed that a single ubiquitin, at least in the form of a non removable, fused ubiquitin, could restore some MVB sorting, but was not as efficient as normal permease ubiquitylation, which involves the addition of several ubiquitin moieties, for MVB sorting (Blondel et al. 2004). The deletion of $B U L 1 / 2$ does not seem to prevent the uracil-induced sorting of Fur4 to the MVB pathway, suggesting that Bul1p/Bul2p may be adaptors involved in the efficient ubiquitylation and sorting of only a subset of Rsp5p transporter substrates. 


\section{Regulated sorting of metal transporters}

Several metal transporters also display regulated sorting during biosynthesis. $S$. cerevisiae takes up iron bound to siderophores by two separate systems, one of which requires the ARN family of siderophore-iron transporters. Arn1p is located in endosome-like intracellular vesicles when produced in the absence of its specific substrate, ferrichrome. If cells are exposed to low concentrations of ferrichrome, Arn $1 p$ is stably redistributed to the plasma membrane (Kim and Yun 2002). Arn1p has two surface binding sites for ferrichrome: a site with low-nanomolar affinity and a site with low-micromolar affinity. Ferrichrome binding to the high-affinity site is required for Arn1p transfer from endosomes to the plasma membrane, as mutations affecting this site also affect the intracellular distribution of Arn1p (Moore et al. 2003). A model has been put forward in which ferrichrome enters cells by fluidphase endocytosis and binds to high-affinity binding sites on endosomal Arn1p, inducing a conformational change and relocalization of the protein to plasma membrane. Arn1p also displays a second type of trafficking regulation: high ferrichrome concentrations induce rapid internalization of the protein at the plasma membrane (Kim and Yun 2002).

Most newly synthesized Smf1p and Smf2p is targeted to the vacuole if manganese is plentiful, and this trafficking involves Bsd2p (Liu et al. 1997), a protein also required for the vacuolar targeting of a misfolded Pmalp variant (Luo and Chang 1997). Manganese starvation triggers the targeting of $\mathrm{Smflp}$ to the plasma membrane, whereas $\mathrm{Smf} 2 \mathrm{p}$ is redistributed to intracellular vesicles (Portnoy et al. 2000). Extensive mutational analysis of Smf1p led to the identification of conserved residues in Smf1p that appear to be required for both Smf1p activity (as defined by complementation of an smflmutation), and Smf1p trafficking to the plasma membrane in cases of manganese depletion (Liu and Culotta 1999a). Thus, for both Arn1p and Smf1p, much is already known about the residues involved in substrate interaction, and substrate-induced regulated trafficking. The potential involvement of ubiquitylation events in these processes was not yet reported.

\section{Recycling of yeast transporters?}

Constant recycling between the plasma membrane and internal compartments is known to be a characteristic feature of a few yeast plasma membrane proteins: the yeast receptor Ste3p (Chen and Davis 2000), and the v-SNARE protein Snc1p (Lewis et al. 2000). These proteins are recycled to the plasma membrane for ligand binding and plasma membrane fusion, respectively, in a process that requires the fusion of endosome-derived vesicles with the trans-Golgi network (TGN), dependent on the small rab Yt6p or the VFT complex (Vps51p, Vps52p...) (Siniossoglou and Pelham 2001). It is unknown whether yeast transporters recycle between internal compartments and the plasma membrane. Permanent recycling was suggested years ago for the $A B C$ transporter Ste6p, which is present in the TGN/endosomes in steady state but functions at the plasma membrane (Kuchler et al. 1993). Recent investigations with end and sec mutants indeed showed that Ste6p can be recycled to the plasma membrane after internalization (Losko et al. 2001).

Several studies have reported the potential recycling of receptors and transporters in class E vps mutants, which would account for the accumulation of these proteins in large amounts at the plasma membrane of these cells (Davis et al. 1993; Li et al. 1999; Forsberg et al. 2001). However, an alternative interpretation of some of these observations is that the observed phenotypes may result from impaired direct vacuolar sorting of transporters synthesized de novo (Forsberg et al. 2001). 
Strong evidence has been obtained for effective recycling of Gap1p after its internalization in class E vps31/brol mutant cells (Nikko et al. 2003). This recycling is impaired in cells mutated in genes (YPT6, TLG1, VPS52) involved in the fusion of endosomederived vesicles with the TGN. Interestingly, such recycling is also defective in vam3 and vam7 mutants impaired in fusion between MVB and the vacuole, raising the possibility that Gap1p is recycled to the cell surface once it has reached the limiting membrane of the vacuole (Nikko et al. 2003). Similar observations have been reported for Fur4p. This permease seems to be stable at the plasma membrane in class E vps mutants under conditions leading to rapid endocytosis in wild-type cells (nitrogen starvation), suggesting internalization followed by recycling. A formal proof of Fur4p recycling from the class E compartment was recently obtained in experiments in which severe stress led to the internalization of GFP-tagged Fur4p and its trapping in the class E compartment. Subsequent release of the stress concerned (in the absence of new permease synthesis) led to recycling of the fluorescent transporter back to the plasma membrane. Strikingly, in this case, recycling did not occur via the Golgi apparatus (no impairment in vps51 $\Delta$ cells) (Bugnicourt and Galan, personal communication). Permeases therefore seem to be able to use several routes for recycling to the plasma membrane in class E vps mutants. It is unknown whether the routes followed by Gap1p and Fur4p in these recycling pathways are similar to or different from the normal sorting of these permeases along the secretory pathway. It is also unclear whether, in wild type cells, rapid transfer to environmental conditions favoring the plasma membrane localization of a given transporter can lead to the recycling of an internalized transporter .

\section{Regulated sorting and recycling of transporters in higher eukaryotes}

Plasma membrane sorting is also regulated for a number of mammalian transporters, and some diseases are associated with deficiencies in such regulation. Two such diseases involve P-type ATPases - the Menkes protein (MNK), and the Wilson's disease protein (WND) both of which are copper transporters, and targets involved in copper disorders. In basal medium, both MNK and WND reside in the TGN, where they transport copper to the lumen of the organelle, providing various copper-dependent enzymes with copper (Petris et al. 2002). Heterologous expression of the MNK gene in yeast can complement the function of the yeast homolog, Ccc2p, which is also located in the TGN (Petris et al. 2002). In conditions of high copper concentration, MNK is rapidly transported from the Golgi apparatus to the plasma membrane, where it is involved in copper efflux. In conditions of high copper concentration, WND is relocalized to cytoplasmic vesicles (Hung et al. 1997). In both cases, copper-induced relocalization occurs independently of new protein synthesis (Petris et al. 1996; Hung et al. 1997). The copper-induced trafficking of MNK and WND presumably switches the function of these ATPases from a nutritional role to a protective role - the export of excess copper from post-Golgi compartments, or across the plasma membrane. Several disease-causing mutations in the MNK and WND genes prevent copper-induced trafficking from the TGN. A conserved feature of all P-type ATPases is the formation of an acyl-phosphate intermediate (Jurgens and Geldner 2002), which occurs as part of the catalytic cycle during cation transport. Extensive mutagenesis, including mutations in the residues affected in patients, has shown that mutations blocking the formation of phosphorylated catalytic intermediates also prevent the copper-induced relocalization of MNK from the TGN. Furthermore, mutations in the phosphatase domain resulting in the hyperphosphorylation of MNK result in constitutive trafficking from the TGN to the plasma membrane. A phosphatase mutation has a similar effect on trafficking in patients with Wilson's disease (Petris et al. 
2002). These data suggest a catalysis-dependent trafficking model, although they do not predict TGN-to-plasma membrane sorting at each catalytic cycle.

In addition to these examples of substrate-induced sorting at the plasma membrane or internal compartments, many transporters in higher eukaryotes display steady-state localization in both endosomal compartments and at the plasma membrane. Rapid changes in surface expression are then achieved by regulation of the relative rates of endocytosis and recycling from endosome compartments to the plasma membrane. In many cases, the proportion of cell-surface versus internal transporters is controlled by hormonal regulation. This is the case for the Glut glucose transporters, the intracellular distribution of which is regulated by insulin. Regulation of this type has been studied in detail (Al-Hasani, this issue). Similarly, the trafficking of the water channel AQP2 is regulated by vasopressin (reviewed in (Royle and Murrell-Lagnado 2003), and Nielsen, this issue). Some ABC transporters in the rat liver are stored in intracellular pools, and presynthesized transporters are sorted from endosomes to the canalicular plasma membrane following stimulation, with cAMP for example (Kipp et al. 2001). In other cases, there is constitutive recycling between endosomes and the plasma membrane, resulting in endosomal and plasma membrane pools of equivalent sizes, as observed for the mammalian Nramp2 metal transporter (Touret et al. 2003), and the $\mathrm{Na}^{+} / \mathrm{H}^{+}$neuronal exchanger NHE5 (Szaszi et al. 2002). Similar situations have also been described for plant transporters. The active, polar transport of auxin through the plant is thought to be due to the coordinated polar distribution of carriers in the plasma membrane: the auxin uptake carrier AUX1 and the auxin efflux carrier PIN1 are distributed apically and basolaterally, respectively. PIN1 undergoes continous cycling between endosomes and the basolateral membrane (reviewed in (Jurgens and Geldner 2002)).

\section{3. 3 Mechanisms and signaling pathways governing the regulated trafficking of transporters}

Although the same machineries seem to be involved in the membrane trafficking and degradation of most, if not all, yeast transporters (e.g. Rsp5p ubiquitin ligase, ESCRT complexes, actin cytoskeleton, etc.), the physiological conditions inducing the downregulation of these transporters or favoring their stabilization at the plasma membrane differ markedly according to transporter considered. Even transporters belonging to the same highly conserved family may display radically different regulation profiles. So, how is the specific control of transporters achieved at the level of membrane trafficking? Recent studies have suggested that substrate recognition, association with lipid rafts, and phosphorylation mediated by protein kinases such as Npr1p are central components determining the fate of transporters.

\section{Role of Nprlp kinase and the TOR signaling pathway}

Studies of amino acid permeases have suggested that the phosphorylation events catalyzed by the Npr1p kinase also play a central role in the regulation of transporter sorting. This kinase (Grenson 1983b; Vandenbol et al. 1987; Vandenbol et al. 1990) prevents Gap1p at the plasma membrane from being sorted to the endocytic and endosome/vacuolar degradation pathways in conditions of nitrogen limitation. It also prevents newly synthesized Gap1p present in the late secretory pathway from being missorted to the vacuole (Fig. 4). If Npr1p is inactivated, Gap1p at the plasma membrane undergoes ubiquitylation, endocytosis and degradation, whereas Gap1p present in the secretory pathway is diverted to the vacuole without passing 
through the plasma membrane (De Craene et al. 2001). Furthermore, Npr1p seems to regulate the Tat $2 p$ and Bap2p permeases in an inverse manner: if cells are transferred from YPD medium to conditions of limiting nitrogen supply, Nprlp simultaneously favors the accumulation of Gap1p at the plasma membrane and promotes the downregulation of Tat $2 p$ and Bap2p. In support of a negative effect of Npr1p on Tat2p permease, the overexpression of NPR1 on rich medium promotes Tat2p inactivation. Furthermore, nprl mutants grown on YPD medium are partially resistant to the negative effect of rapamycin on Tat $2 p$ activity (Schmidt et al. 1998). However, the mechanisms underlying the inverse control of the fate of Gap1p, Tat2p and Bap2p by Npr1p remain unknown. Gap1p may be phosphorylated, even in an nprl mutant (De Craene et al. 2001), but this does not rule out the possibility that Npr1p is involved in the phosphorylation of the permease in normal conditions. Glutathione-Stransferase fused to the $\mathrm{N}$ - and C-terminal cytosolic tails of Bap2p was recently shown to undergo Npr1p-dependent phosphorylation in response to rapamycin (conditions promoting Npr1 activity), suggesting that Npr1p directly phosphorylates the permeases via their tails (Omura and Kodama 2003).

The apparently key role of Npr1p in the nitrogen-regulated sorting of amino acid permeases raises a further question: how is Nprlp regulated by nitrogen? Nprlp is phosphorylated in conditions in which nitrogen is abundant (in which Nprlp is apparently inactive) and is dephosphorylated if cells are transferred to poor nitrogen conditions or if rapamycin is added to the medium (i.e., when Nprlp is active). This dephosphorylation requires Tap42p, a phosphatase under control of the TOR signaling pathway (Beck and Hall 1999). Tor $1 p$ and Tor $2 p$ (targets of rapamycin) are phosphatidylinositol kinase-related protein kinases controlling multiple cellular functions in response to nutrients (Crespo and Hall 2002). Recent studies have shown that the TOR proteins are subunits of two large protein complexes (Loewith et al. 2002). Both these complexes include Lst8p, the partial deficiency of which leads to the loss of Gap1p activity (Roberg et al. 1997a; Roberg et al. 1997b). It was proposed that Npr1p is controlled by phosphorylation via the TOR pathway, which is itself regulated by nutrients (Beck and Hall 1999). However, it remains unclear how nutrients control TOR, both in yeast and higher organisms (Crespo and Hall 2002; Manning and Cantley 2003). Starting from the observation that the internal pool of amino acids has a strong influence on Gap1p sorting (Chen \& Kaiser, 2002), C. Kaiser and coworkers proposed an alternative model. According to this model, the influence of TOR and rapamycin on the regulation of Gap1p is indirect, with TOR affecting only the internal pool of amino acids through its effects on transcription of the genes involved in amino-acid biosynthesis. This internal pool of amino acids then controls the fate of Gap1p: Gap1p is downregulated if the internal pool of amino acids is large and sorted to the plasma membrane if this pool is small (Chen and Kaiser 2003). However, the means by which the amino acid pool controls Gap1 trafficking remain unknown. Internal amino acids may inhibit Npr1p activity. Alternatively, they may bind to Gap1p, thereby inducing a conformational change promoting sorting of the permease to the MVB/vacuolar degradation pathway. However, this second model must also take into account the observation that a lack of Npr1p kinase is sufficient to induce Gap1p degradation (De Craene et al. 2001). 


\section{Role of substrate binding and association with lipid rafts}

There is growing evidence that the binding of substrates to transporters plays a central role in their controlled membrane trafficking. For instance, we have previously reported that a mutant form of Fur4p, unable to recognize its substrate uracil even though it reaches the cell surface normally (Urban-Grimal et al. 1995), is insensitive to uracil-induced endocytosis (Séron et al. 1999) and sorting to the vacuole (Blondel et al. 2004). This observation suggests a model in which Fur4p exists in two conformations according to the relative abundance of uracil: one amenable (when uracil is abundant) and the other insensitive (when uracil is present in low concentrations) to ubiquitylation. As the phosphorylation of Fur4p is a prerequisite for its ubiquitylation at the plasma membrane (Marchal et al. 1998), internal uracil may act by altering the phosphorylation status of the permease. This would modulate the sensitivity of the permease to ubiquitylation, unmasking Fur4p domains interacting directly or indirectly with Rsp5p domains. Intracellular uracil is also the critical signal triggering the direct sorting of newly synthesized Fur4p to the endosomal pathway, ultimately leading to its Rsp5p-dependent ubiquitylation and MVB sorting, an event that does not require prior Fur4p phosphorylation, at least as far as the PEST sequence is concerned (Blondel et al. in press). Other possibilities could be suggested in this case, such as a substrate-induced conformational change leading to segregation into specific lipid domains, required for Rsp5p-dependent ubiquitylation. Here too, the relevant substrate/enzyme mode of interaction remains to be defined.

A link between lipid-raft association and substrate availability is also suggested by recent work on control of the sorting of the Tat $2 p$ permease by one of its main substrates, tryptophan (Umebayashi and Nakano 2003) (see 17.3.2). These data are consistent with Tat2p binding to tryptophan (when tryptophan is abundant), leading to its dissociation from lipid rafts and sorting to the MVB/vacuole in a ubiquitylation-dependent manner. For instance, tryptophan may cause Tat2p to undergo a conformational change incompatible with raft association. The overproduction of Npr1p may inhibit Tat2p activity (Schmidt et al. 1998) by direct phosphorylation of the permease, thereby inducing a conformational change mimicking that normally adopted on high-tryptophan medium.

The best illustration of the role of substrate binding in controlling the membrane trafficking of transporters is probably that of the Arn1p and Arn3p ferrichrome (FC) transporters (Kim and Yun 2002; Moore et al. 2003) (see 17.3. 2). Only the substrates of each Arn protein promote their sorting from endosomes to the plasma membrane. Furthermore, in the model proposed by $\mathrm{C}$. Philpott and coworkers, FC enters the cell by fluid-phase endocytosis and interacts with Arn domains exposed to the lumen of the early endosome. This binding leads the Arn proteins to change conformation and induces their sorting to the plasma membrane. The possible involvement of an association between Arn proteins and lipid rafts in the sorting of these proteins to the cell surface has not been demonstrated. The proposed model also raises the general question of the role of fluid-phase endocytosis in the substratemediated controlled sorting of transporters present in internal compartments. The binding of FC to a second, lower-affinity binding site exposed by Arn1p at the plasma membrane may induce a second conformational change, promoting the endocytosis of Arn1p (Moore et al. 2003). 


\section{Conclusion}

Most membrane transporters are tightly regulated at the membrane trafficking level and studies carried out over the last ten years have generated a considerable body of novel data, revealing some of the general features of the underlying mechanisms. It has been demonstrated that the covalent attachment of ubiquitin to the cytosolic regions of transporters is an essential prerequisite for the sorting of transporters located at the plasma membrane to endocytic vesicles and for the sorting of transporters present in endosomal compartments to the MVB/vacuolar degradation pathway (Fig. 1 and 4). The ubiquitin ligase Rsp5p, acting together or not with its Bul partners, mediates the ubiquitylation of proteins at the plasma membrane (Rotin et al. 2000; Hicke and Dunn 2003) and also in the Golgi to MVB pathway (Helliwell et al. 2001; Soetens et al. 2001; Umebayashi and Nakano 2003; Blondel et al. 2004). The main components of the cellular machineries involved in the recognition and sorting of ubiquitylated cargos have now been identified. Identification of the upstream molecular events triggering the ubiquitylation of specific transporters in response to appropriate environmental stimuli is a key aim of current research. Evidence is accumulating that at least three events are linked to the likelihood of a transporter undergoing ubiquitylation: association of the protein with specific lipid environments, possible phosphorylation by protein kinases, and specific binding of the transporter to its own substrates. It is tempting to suggest that transporters may adopt different conformational states, dictating their association with specific lipid environments and their probability of being ubiquitylated. The conformation adopted by the transporters may, in turn, be modulated by the binding of their own substrates, and possibly also by phosphorylation. Transporters may also have distinct substrate-binding domains, oriented either towards the extracellular medium (i.e., the lumen of compartments if the protein is internal) or the cytosol. These simple molecular mechanisms would readily generate a large diversity of controls adapted to the physiological roles of each transporter. Future work will thus need to improve our understanding of the complex relationships between the processes involved and to determine whether the conclusions drawn for yeast proteins also apply to the transporters of mammalian cells, which display as well substrate-induced trafficking regulation.

\section{Acknowledgements}

We thank the members of André's and Haguenauer-Tsapis's labs for fruitful discussions and critical reading of this manuscript. We are grateful to Alex Edelman \& Associates for editorial assistance. The work performed in our laboratories is supported by an EU program (EFFEXPORT, contract QLRT-2001-00533). Work of the laboratory of R. HaguenauerTsapis is supported by the Centre National de la Recherche Scientifique, the Universities Paris 6 and Paris 7, and a grant from the Association pour la recherche contre le Cancer (ARC, grant no. 5681), and work of the laboratory of B. André is supported by a grant FRSM 3.4597.00 for Medical Scientific Research, Belgium. 


\section{Figure legends}

Fig.1. Intracellular trafficking of a transporter along the secretory and endocytic pathways. After ER insertion, misfolded transporters undergo ERAD and proteasomal degradation. Correctly folded transporters are packaged in COPII vesicles, with the aid of chaperones, such as Shr3p in the case of aminoacid permeases. ER to Golgi and Golgi to plasma membrane trafficking is dependent on a number of $S E C$ genes. Whether trafficking from Golgi to plasma membrane occurs directly via secretory vesicles, or via late endosomes and then secretory vesicles is still unknown. Many transporters undergo phosphorylation $(\mathrm{P})$, in some instances a plasma membrane event dependent of Yck1/2 kinases. Specific physiological signals trigger Rsp5p-dependent ubiquitylation (ub), also dependent of the Bul proteins, as described for some transporters. This modification acts as a signal triggering transporters internalization followed by targeting to early endosomes (EE). At the late endosome/MVB, transporters undergo sorting to internal vesicles, an event dependent on Vps class E proteins, followed by Doa4p-dependent deubiquitylation. Vam-dependent fusion of MVB with the vacuole leads to delivery of internal MVB vesicles to the vacuolar lumen accompanied by transporters degradation by vacuolar proteases.

Fig. 2. Type of ubiquitylation of yeast transporters. Ubiquitin carries 11 Lys, some of which notably Lys29, 48 and 63.Lys, act as target Lys for linkage to other ubiquitin residues. Lys residues in target proteins can receive a single ubiquitin (monoubiquitylation), or chains of ubiquitin moities, linked either via ubiquitin Lys48, or Lys63. Lys48-linked ubiquitin chains, at least four residues long target proteins for proteasomal degradation. Some yeast plasma membrane transporters were described to display primarily monoubiquitylation. Fur4p and Gap1p receive on their two target Lys two-three ubiquitin residues linked via ubiquitin Lys63. Other transporters appear to display polyubiquitylation, with undetermined type of ubiquitin linkage, notably during direct Golgi to vacuolar targeting.

Fig. 3. Substrate-induced endocytosis of the uracil permease Fur4p: ubiquitin-dependent, Rsp5p-dependent plasma membrane internalization and MVB sorting.

GFP-tagged Fur4p or a variant Fur4p mutated in its two target Lys (Lys38, 41) fused or not to a variant ubiquitin lacking Lys acceptor sites (Ub) were expressed in wild type (WT) or $n p i$ (npil/rsp5) mutant cells. The fate of presynthesized permease was followed before and two hours $(2 \mathrm{H})$ after addition of uracil in the medium by visualizing GFP fluorescence. Vacuolar lumen was visualized using a dye, CMAC. Fur4-GFP undergoes uracil-induced endocytosis in wild type cells: fluorescence disappears from the plasma membrane, and is recovered in the vacuole lumen (low rate of degradation of the GFP tag). Internalization is impaired by mutation of Fur4 target Lys, or by the npil mutation in RSP5 gene. In frame fusion of ubiquitin lacking its own target Lys restores some internalization, and triggers partial direct vacuolar sorting, but MVB sorting is not entirely efficient, and both neosynthesized permease and internalized permease are partially retained at the vacuolar membrane.

This represents selected data from experiments reported in (Blondel et al. 2004), with permission of the Editor.

Fig. 4. Direct vacuolar targeting of plasma membrane transporters. A number of physiological (nutrient changes, excess substrate), or pathological situations (mutation in transporters, or in Npr1p kinase, deficiencies in lipid metabolism) lead to direct Golgi to vacuolar sorting of transporters bypassing plasma membrane delivery. These transporters undergo Rsp5p-dependent ubiquitylation, in some cases with the help of the Bul proteins, possibly required for polyubiquitylation. The route followed is either first Golgi to early 
endosome (EE), or Golgi to late endosome/MVB, where ubiquitylation results in sorting into MVB vesicles. MVB fusion with the vacuole is followed by vacuolar degradation.

\section{References}

Abe F, lida $\mathrm{H}$ (2003) Pressure-induced differential regulation of the two tryptophan permeases Tat 1 and Tat2 by ubiquitin ligase Rsp5 and its binding proteins Bul1 and Bul2. Mol Cell Biol 23: 7566-7584

Abriel H, Kamynina E, Horisberger J-D, Staub O (2000) Regulation of the cardiac voltagegated $\mathrm{Na}+$ channel $(\mathrm{rH} 1)$ by the ubiquitin-protein ligase Nedd4. FEBS Lett 466: 377380

Aguilar RC, Watson HA, Wendland B (2003) The yeast Epsin Ent1 is recruited to membranes through multiple independent interactions. J Biol Chem 278: 1073710743

Ahmad M, Bussey $\mathrm{H}$ (1988) Topology of membrane insertion in vitro and plasma membrane assembly in vivo of the yeast arginine permease. Mol Microbiol 2: 627-635

Amerik AY, Nowak J, Swaminathan S, Hochstrasser M (2000) The Doa4 deubiquitinating enzyme is functionally linked to the vacuolar protein-sorting and endocytic pathways. Mol Biol Cell 11: 3365-3380

Arvan P, Zhao X, Ramos-Castaneda J, Chang A (2002) Secretory pathway quality control operating in Golgi, plasmalemmal, and endosomal systems. Traffic 3: 771-780.

Baggett JJ, Wendland B (2001) Clathrin function in yeast endocytosis. Traffic 2: 297-302.

Bagnat M, Chang A, Simons K (2001) Plasma membrane proton ATPase pma1p requires raft association for surface delivery in yeast. Mol Biol Cell 12: 4129-4138.

Beaudenon SL, Huacani MR, Wang G, McDonnell DP, Huibregtse JM (1999) Rsp5 ubiquitin-protein ligase mediates DNA damage-induced degradation of the large subunit of RNA polymerase II in Saccharomyces cerevisiae. Mol Cell Biol 19: 69726979

Beck T, Hall MN (1999) The TOR signalling pathway controls nuclear localization of nutrientregulated transcription factors. Nature 402: 689-692

Beck T, Schmidt A, Hall MN (1999) Starvation induces vacuolar targeting and degradation of the tryptophan permease in yeast. J Cell Biol 146: 1227-1238

Bilodeau PS, Urbanowski JL, Winistorfer SC, Piper RC (2002) The Vps27p Hse1p complex binds ubiquitin and mediates endosomal protein sorting. Nat Cell Biol 4: 534-539

Bilodeau PS, Winistorfer SC, Kearney WR, Robertson AD, Piper RC (2003) Vps27-Hse1 and ESCRT-I complexes cooperate to increase efficiency of sorting ubiquitinated proteins at the endosome. J Cell Biol 163: 237-243

Blondel MO, Morvan J, Dupré S, Urban-Grimal D, Haguenauer-Tsapis R, Volland C (2004) Direct sorting of the yeast uracil permease to the endosomal system is controlled by uracil availibility and Rsp5p-dependent ubiquitylation. Mol Biol Cell 15: in press

Bonifacino JS, Traub LM (2003) Signals for sorting of transmembrane proteins to endosomes and lysosomes. Annu Rev Biochem 72: 395-447

Brondijk TH, van der Rest ME, Pluim D, de Vries Y, Stingl K, Poolman B, Konings WN (1998) Catabolite inactivation of wild-type and mutant maltose transport proteins in Saccharomyces cerevisiae. J Biol Chem 273: 15352-15357

Buckley KM, Melikian HE, Provoda CJ, Waring MT (2000) Regulation of neuronal function by protein trafficking: a role for the endosomal pathway. J Physiol 525 Pt 1: 11-19

Buziol S, Becker J, Baumeister A, Jung S, Mauch K, Reuss M, Boles E (2002) Determination of in vivo kinetics of the starvation-induced Hxt5 glucose transporter of Saccharomyces cerevisiae. FEMS Yeast Res 2: 283-291

Chang A, Cheang S, Espanel X, Sudol M (2000) Rsp5 WW domains interact directly with the carboxyl-terminal domain of RNA polymerase II. J Biol Chem 275: 20562-20571 
Chang A, Slayman CW (1991) Maturation of the yeast plasma membrane [H+]ATPase involves phosphorylation during intracellular transport. J Cell Biol 115: 289-295

Chen EJ, Kaiser CA (2003) LST8 negatively regulates amino acid biosynthesis as a component of the TOR pathway. J Cell Biol 161: 333-347

Chen L, Davis NG (2000) Recycling of the yeast a-factor receptor. J Cell Biol 151: 731-738

Crespo JL, Hall MN (2002) Elucidating TOR signaling and rapamycin action: lessons from Saccharomyces cerevisiae. Microbiol Mol Biol Rev 66: 579-591

D'Hondt K, Heese-Peck A, Riezman H (2000) Protein and lipid requirements for endocytosis. Annu Rev Genet 34: 255-295

Dancis A, Haile D, Yuan DS, Klausner RD (1994) The Saccharomyces cerevisiae copper transporter protein (Ctr1p). J Biol Chem 269: 25660-25667

David D, Sundarababu S, Gerst JE (1998) Involvement of long chain fatty acid elongation in the trafficking of secretory vesicles in yeast. J Cell Biol 143: 1167-1182

Davis NG, Horecka JL, Sprague JGF (1993) Cis- and trans- acting functions required for endocytosis of the yeast pheromone receptors. J Cell Biol 122: 53-65

De Craene JO, Soetens O, André B (2001) The Npr1 kinase controls biosynthetic and endocytic sorting of the yeast Gap1 permease. J Biol Chem 276: 43939-44348

Debonneville C, Flores SY, Kamynina E, Plant PJ, Tauxe C, Thomas MA, Munster C, Chraibi A, Pratt JH, Horisberger JD, Pearce D, Loffing J, Staub O (2001) Phosphorylation of Nedd4-2 by Sgk1 regulates epithelial $\mathrm{Na}(+)$ channel cell surface expression. EMBO J 20: 7052-7059.

Decottignies A, Owsianik G, Ghislain M (1999) Casein Kinase-I-dependent Phosphorylation and Stability of the Yeast Multidrug Transporter Pdr5p. J Biol Chem 274: 3713937146

Dunn R, Hicke L (2001) Domains of the Rsp5 ubiquitin-protein ligase required for receptormediated and fluid-phase endocytosis. Mol Biol Cell. 12: 421-435

Dupre S, Haguenauer-Tsapis R (2003) Raft partitioning of the yeast uracil permease during trafficking along the endocytic pathway. Traffic 4: 83-96

Dupré S, Haguenauer-Tsapis R (2001) Deubiquitination step in the endocytic pathway of yeast plasma membrane proteins: crucial role of Doa4p ubiquitin isopeptidase. Mol Cell Biol 21: 4482-4494.

Egner R, Kuchler K (1996) The yeast multidrug transporter Pdr5 of the plasma membrane is ubiquitinated prior to endocytosis and degradation in the vacuole. FEBS Lett 378 : 177-181

Egner R, Mahé Y, Pandjaitan R, Kuchler K (1995) Endocytosis and vacuolar degradation of the plasma membrane-localized Pdr5 ATP-binding cassette multidrug transporter in Saccharomyces cerevisiae. Mol Cell Biol 15: 5879-5887

Ferro-Novick S, Novick P, Field C, Schekman R (1984) Yeast secretory mutants that block the formation of active cell surface enzymes. J Cell Biol 98: 35-43

Forsberg H, Hammar M, Andreasson C, Moliner A, Ljungdahl PO (2001) Suppressors of ssy1 and ptr3 null mutations define novel amino acid sensor-independent genes in Saccharomyces cerevisiae. Genetics 158: 973-988

Gagny B, Wiederkehr A, Dumoulin P, Winsor B, Riezman H, Haguenauer-Tsapis R (2000) A novel EH domain protein of Saccharomyces cerevisiae involved in endocytosis. J Cell Sci 113: 3309-3319

Gajewska B, Kaminska J, Jesionowska A, Martin NC, Hopper AK, Zoladek T (2001) WW domains of Rsp5p define different functions: determination of roles in fluid phase and uracil permease endocytosis in Saccharomyces cerevisiae. Genetics 157: 91-101.

Galan J-M, Haguenauer-Tsapis R (1997) Ubiquitin Lys63 is involved in ubiquitination of a yeast plasma membrane protein. EMBO J 16: 5847-5854

Galan JM, Cantegrit B, Garnier C, Namy O, Haguenauer-Tsapis R (1997) "ER degradation" of a mutant yeast plasma membrane protein by the ubiquitin-proteasome pathway.

FASEB J 12: 315-323 
Galan JM, Moreau V, André B, Volland C, Haguenauer-Tsapis R (1996) Ubiquitination mediated by the Npi1p/Rsp5p ubiquitin-protein ligase is required for endocytosis of the yeast uracil permease. J Biol Chem 271: 10946-10952

Garnier C, Blondel MO, Hagenauer-Tsapis R (1996) Membrane topology of the yeast uracil permease. Mol Microbiol 21: 1061-1073

Gilstring CF, Ljungdahl PO (2000) A method for determining the in vivo topology of yeast polytopic membrane proteins demonstrates that Gap1p fully integrates into the membrane independently of Shr3p. J Biol. Chem 275: 31488-31495

Giorgino F, de Robertis O, Laviola L, Montrone C, Perrini S, McCowen KC, Smith RJ (2000) The sentrin-conjugating enzyme mUbc9 interacts with GLUT4 and GLUT1 glucose transporters and regulates transporter levels in skeletal muscle cells. Proc Natl Acad Sci U S A 97: 1125-1130

Gitan RS, Eide DJ (2000) Zinc-regulated Ubiquitin Conjugation Signals Endocytosis of the yeast ZRT1 Zinc Transporter. Biochem J 346: 329-336

Gitan RS, Luo H, Rodgers J, Broderius M, Eide D (1998) Zinc-induced inactivation of the yeast $Z R T 1$ zinc transporter occurs through endocytosis and vacuolar degradation. $\mathrm{J}$ Biol Chem 273: 28617-28624

Gitan RS, Shababi M, Kramer M, Eide DJ (2003) A cytosolic domain of the yeast Zrt1 zinc transporter is required for its post-translational inactivation in response to zinc and cadmium. J Biol Chem

Glickman MH, Ciechanover A (2002) The ubiquitin-proteasome proteolytic pathway: destruction for the sake of construction. Physiol Rev 82: 373-428.

Goossens A, de La Fuente N, Forment J, Serrano R, Portillo F (2000) Regulation of yeast $\mathrm{H}(+)$-ATPase by protein kinases belonging to a family dedicated to activation of plasma membrane transporters. Mol Cell Biol 20: 7654-7661

Graschopf A, Stadler JA, Hoellerer MK, Eder S, Sieghardt M, Kohlwein SD, Schweyen RJ (2001) The yeast plasma membrane protein Alr1 controls Mg2+ homeostasis and is subject to Mg2+-dependent control of its synthesis and degradation. J Biol Chem 276: 16216-16222.

Grenson M (1983a) Inactivation-reactivation process and repression of permease formation regulate several ammonia-sensitive permeases in the yeast Saccharomyces cerevisiae. Eur J Biochem 133: 135-139

Grenson M (1983b) Study of the positive control of the general amino-acid permease and other ammonia-sensitive uptake systems by the product of the NPR1 gene in the yeast Saccharomyces cerevisiae. Eur J Biochem 133: 141-144

Grenson M (1992) Amino acid transporters in yeast: structure, function and regulation. In Pont, D (ed.), Molecular Aspects of Transport Proteins. Elsevier Science Publishers B. V, Amsterdam, pp. 219-245.

Grenson M, Hou C, Crabeel M (1970) Multiplicity of the amino acid permeases in Saccharomyces cerevisiae. IV. Evidence for a general amino acid permease. J Bacteriol 103: 770-777

Gurunathan S, David D, Gerst J (2002) Dynamin and clathrin are required for the biogenesis of a distinct class of secretory vesicles in yeast. EMBO J 21: 602-614

Hampton RY (2002) ER-associated degradation in protein quality control and cellular regulation. Curr Opin Cell Biol 14: 476-482

Harsay E, Bretscher A (1995) Parallel secretory pathways to the cell surface in yeast. J Cell Biol 131: 297-310

Harsay E, Schekman R (2002) A subset of yeast vacuolar protein sorting mutants is blocked in one branch of the exocytic pathway. J Cell Biol 156: 271-285.

Hein C, André B (1997) A C-terminal di-leucine motif and nearby sequences are required for $\mathrm{NH} 4(+)$-induced inactivation and degradation of the general amino acid permease, Gap1p, of Saccharomyces cerevisiae. Mol Microbiol 24: 607-616 
Hein C, Springael JY, Volland C, Haguenauer-Tsapis R, André B (1995) NPI1, an essential yeast gene involved in induced degradation of Gap1 and Fur4 permeases, encodes the Rsp5 ubiquitin-protein ligase. Mol Microbiol 18: 77-87

Helliwell SB, Losko S, Kaiser CA (2001) Components of a ubiquitin ligase complex specify polyubiquitination and intracellular trafficking of the general amino acid permease. $\mathrm{J}$ Cell Biol 153: 649-662.

Hicke L, Dunn R (2003) Regulation of membrane protein transport by ubiquitin and ubiquitinbinding proteins. Annu Rev Cell Dev Biol 19: 141-172

Hicke L, Zanolari B, Riezman H (1998) Cytoplasmic tail phosphorylation of the alpha-factor receptor is required for its ubiquitination and internalization. J Cell Biol 141: 349-358

Hitchcock AL, Auld K, Gygi SP, Silver PA (2003) A subset of membrane-associated proteins is ubiquitinated in response to mutations in the endoplasmic reticulum degradation machinery. Proc Natl Acad Sci U S A 100: 12735-12740

Hoppe T, Matuschewski K, Rape M, Schlenker S, Ulrich HD, Jentsch S (2000) Activation of a membrane-bound transcription factor by regulated ubiquitin/proteasomedependent processing. Cell 102: 577-586.

Horak $J$ (2003) The role of ubiquitin in down-regulation and intracellular sorting of membrane proteins: insights from yeast. Biochim Biophys Acta 1614: 139-155

Horak J, Wolf DH (1997) Catabolite inactivation of the galactose transporter in the yeast Saccharomyces cerevisiae: ubiquitination, endocytosis, and degradation in the vacuole. J Bacteriol 179: 1541-1549

Horak J, Wolf DH (2001) Glucose-induced monoubiquitination of the Saccharomyces cerevisiae galactose transporter is sufficient to signal its internalization. J Bacteriol 183: 3083-3088.

Huang KM, D'Hondt K, Riezman H, Lemmon SK (1999) Clathrin functions in the absence of heterotetrameric adaptors and AP180-related proteins in yeast. EMBO J 18: 38973908

Huh WK, Falvo JV, Gerke LC, Carroll AS, Howson RW, Weissman JS, O'Shea EK (2003) Global analysis of protein localization in budding yeast. Nature 425: 686-691

Hung IH, Suzuki M, Yamaguchi Y, Yuan DS, Klausner RD, Gitlin JD (1997) Biochemical characterization of the Wilson disease protein and functional expression in the yeast Saccharomyces cerevisiae. J Biol Chem 272: 21461-21466

Ikonen E (2001) Roles of lipid rafts in membrane transport. Curr Opin Cell Biol 13: 470-477.

Iraqui I, Vissers S, Bernard F, de Craene JO, Boles E, Urrestarazu A, André B (1999) Amino acid signaling in Saccharomyces cerevisiae: a permease-like sensor of external amino acids and F-Box protein Grr1p are required for transcriptional induction of the AGP1 gene, which encodes a broad-specificity amino acid permease. Mol Cell Biol 19: 989-1001

Ishida S, Lee J, Thiele DJ, Herskowitz I (2002) Uptake of the anticancer drug cisplatin mediated by the copper transporter Ctr1 in yeast and mammals. Proc Natl Acad Sci U S A 99: 14298-14302

Jauniaux JC, Grenson M (1990) GAP1, the general amino acid permease gene of Saccharomyces cerevisiae. Nucleotide sequence, protein similarity with the other bakers yeast amino acid permeases, and nitrogen catabolite repression. Eur $\mathrm{J}$ Biochem 190: 39-44

Jund R, Weber E, Chevallier MR (1988) Primary structure of the uracil transport protein of Saccharomyces cerevisiae. Eur J Biochem 171: 417-424

Jurgens G, Geldner N (2002) Protein secretion in plants: from the trans-Golgi network to the outer space. Traffic 3: $605-613$

Kamynina E, Staub O (2002) Concerted action of ENaC, Nedd4-2, and Sgk1 in transepithelial $\mathrm{Na}(+)$ transport. Am J Physiol Renal Physiol 283: F377-387 
Kaouass M, Gamache I, Ramotar D, Audette M, Poulin R (1998) The spermidine transport system is regulated by ligand inactivation, endocytosis, and by the Npr1p Ser/Thr protein kinase in Saccharomyces cerevisiae. J Biol Chem 273: 2109-2117

Katzmann D, Odorizzi G, Emr S (2002) Receptor downregulation and multivesicular-body sorting. Nat Rev Mol Cell Biol 3: 893-905

Katzmann DJ, Babst M, Emr SD (2001) Ubiquitin-dependent sorting into the multivesicular body pathway requires the function of a conserved endosomal protein sorting complex, ESCRT-I. Cell 106: 145-155

Kim Y, Yun C (2002) Ferrichrome induces endosome to plasma membrane cycling of the ferrichrome transporter, Arn1p, in

Saccharomyces cerevisiae. EMBO J 21: 3632-3642.

Kipp H, Pichetshote N, Arias IM (2001) Transporters on demand: intrahepatic pools of canalicular ATP binding cassette transporters in rat liver. J Biol Chem 276: 7218-7224

Kohler JR (2003) Mos10 (Vps60) is required for normal filament maturation in Saccharomyces cerevisiae. Mol Microbiol 49: 1267-1285

Kolling R (2002) Mutations affecting phosphorylation, ubiquitination and turnover of the ABCtransporter Ste6. FEBS Lett 531: 548-552

Kolling R, Hollenberg CP (1994a) The ABC-transporter Ste6 accumulates in the plasma membrane in a ubiquitinated form in endocytosis mutants. EMBO J 13: 3261-3271

Kolling R, Hollenberg CP (1994b) The first hydrophobic segment of the ABC-transporter, Ste6, functions as a signal sequence. FEBS Lett 351: 155-158

Kolling R, Losko S (1997) The linker region of the ABC-transporter Ste6 mediates ubiquitination and fast turnover of the protein. EMBO J 16: 2251-2261

Kostova Z, Wolf DH (2003) For whom the bell tolls: protein quality control of the endoplasmic reticulum and the ubiquitin-proteasome connection. EMBO J 22: 2309-2317

Krampe S, Boles E (2002) Starvation-induced degradation of yeast hexose transporter Hxt7p is dependent on endocytosis, autophagy and the terminal sequences of the permease. FEBS Lett 513: 193-196

Krampe S, Stamm O, Hollenberg CP, Boles E (1998) Catabolite inactivation of the high affinity hexose transporters Hxt6 and Hxt7 of Saccharomyces cerevisiae occurs in the vacuole after internalization by endocytosis. FEBS lett 441: 343-347

Kranz A, Kinner A, Kolling R (2001) A family of small coiled-coil-forming proteins functioning at the late endosome in yeast. Mol Biol Cell 12: 711-723

Kruckeberg AL, Ye L, Berden JA, van Dam K (1999) Functional expression, quantification and cellular localization of the Hxt2 hexose transporter of Saccharomyces cerevisiae tagged with the green fluorescent protein. Biochem J 339 ( Pt 2): 299-307

Kuchler K, Dohlman HG, Thorner J (1993) The a-factor transporter (STE6 gene product) and cell polarity in the yeast Saccharomyces cerevisiae. J Cell Biol 120: 1203-1215

Lai K, Bolognese CP, Swift S, McGraw P (1995) Regulation of inositol transport in Saccharomyces cerevisiae involves inositol-induced changes in permease stability and endocytic degradation in the vacuole. J Biol Chem 270: 2525-2534

Lai K, McGraw P (1994) Dual control of inositol transport in Saccharomyces cerevisiae by irreversible inactivation of permease and regulation of permease synthesis by INO2, INO4, and OPI1. J Biol Chem 269: 2245-2251

Lee VH (2000) Membrane transporters. Eur J Pharm Sci 11 Suppl 2: S41-S50

Lewis MJ, Nichols BJ, Prescianotto-Baschong C, Riezman H, Pelham HR (2000) Specific retrieval of the exocytic SNARE Snc1p from early yeast endosomes. Mol Biol Cell 11: 23-38

Li Y, Kane T, Tipper C, Spatrick P, Jenness DD (1999) Yeast mutants affecting possible quality control of plasma membrane proteins. Mol Cell Biol 19: 3588-3599

Liu XF, Culotta VC (1999a) Mutational analysis of Saccharomyces cerevisiae Smf1p, a member of the Nramp family of metal transporters. J Mol Biol 289: 885-891 
Liu XF, Culotta VC (1999b) Post-translation control of Nramp metal transport in yeast. Role of metal ions and the BSD2 gene. J Biol Chem 274: 4863-4868

Liu XF, Supek F, Nelson N, Culotta VC (1997) Negative control of heavy metal uptake by the Saccharomyces cerevisiae BSD2 gene. J Biol Chem 272: 11763-11669

Loayza D, Tam A, Schmidt WK, Michaelis S (1998) Ste6p mutants defective in exit from the endoplasmic reticulum (ER) reveal aspects of an ER quality control pathway in Saccharomyces cerevisiae. Mol Biol Cell 9: 2767-2784

Loewith R, Jacinto E, Wullschleger S, Lorberg A, Crespo JL, Bonenfant D, Oppliger W, Jenoe $P$, Hall MN (2002) Two TOR complexes, only one of which is rapamycin sensitive, have distinct roles in cell growth control. Mol Cell Biol 10: 457-468

Losko S, Kopp F, Kranz A, Kolling R (2001) Uptake of the ATP-binding cassette (ABC) transporter Ste6 into the yeast vacuole is blocked in the doa4 Mutant. Mol Biol Cell 12: $1047-1059$

Lucero P, Lagunas R (1997) Catabolite inactivation of the yeast maltose transporter requires ubiquitin-ligase npi1/rsp5 and ubiquitin-hydrolase npi2/doa4. FEMS Microbiol Lett 147: 273-277

Lucero P, Moreno E, Lagunas R (2002) Catabolite inactivation of the sugar transporters in Saccharomyces cerevisiae is inhibited by the presence of a nitrogen source. FEMS Yeast Res 1: 307-314

Lucero P, Penalver E, Vela L, Lagunas R (2000) Monoubiquitination is sufficient to signal internalization of the maltose transporter in Saccharomyces cerevisiae. J Bacteriol 182: 241-243.

Luo WJ, Chang A (1997) Novel genes involved in endosomal traffic in yeast revealed by supression of a targeting-defective plasma membrane ATPase mutant. J Cell Biol 138: $731-746$

Malkus P, Jiang F, Schekman R (2002) Concentrative sorting of secretory cargo proteins into COPII-coated vesicles. J Cell Biol 159: 915-921

Manning BD, Cantley LC (2003) Rheb fills a GAP between TSC and TOR. Trends Biochem Sci 28: 573-576

Marchal C, Haguenauer-Tsapis R, Urban-Grimal D (1998) A PEST-like sequence mediates phosphorylation and efficient ubiquitination of the yeast uracil permease. Mol Cell Biol 18: 314-321

Marchal C, Haguenauer-Tsapis R, Urban-Grimal D (2000) Casein kinase I-dependent phosphorylation within a PEST sequence and ubiquitination at nearby lysines, signal endocytosis of yeast uracil permease. J Biol Chem 275: 23608-23614

Marini AM, André B (2000) In vivo N-glycosylation of the mep2 high-affinity ammonium transporter of Saccharomyces cerevisiae reveals an extracytosolic $\mathrm{N}$-terminus. Mol Microbiol 38: 552-564

Martinez P, Zvyagilskaya R, Allard P, Persson BL (1998) Physiological regulation of the derepressible phosphate transporter in Saccharomyces cerevisiae. J Bacteriol 180: 2253-2256

Mason DL, Michaelis S (2002) Requirement of the N-terminal extension for vacuolar trafficking and transport activity of yeast Ycf1p, an ATP-binding cassette transporter. Mol Biol Cell 13: 4443-4455

Medintz I, Jiang H, Michels C (1998) The role of ubiquitin conjugation in glucose-induced proteolysis of $S$. cerevisiae maltose permease. J Biol Chem 273: $34454-34462$

Medintz I, Wang X, Hradek T, Michels CA (2000) A PEST-like sequence in the N-terminal cytoplasmic domain of Saccharomyces maltose permease is required for glucoseinduced proteolysis and rapid inactivation of transport activity. Biochemistry 182: 4518-4526

Miller EA, Beilharz TH, Malkus PN, Lee MC, Hamamoto S, Orci L, Schekman R (2003) Multiple cargo binding sites on the COPII subunit Sec24p ensure capture of diverse membrane proteins into transport vesicles. Cell 114: 497-509 
Moore RE, Kim Y, Philpott CC (2003) The mechanism of ferrichrome transport through Arn1p and its metabolism in Saccharomyces cerevisiae. Proc Natl Acad Sci U S A 100: $5664-5669$

Mukherjee S, Ghosh RN, Maxfield FR (1997) Endocytosis. Physiol Rev 77: 759-803

Murillas R, Simms KS, Hatakeyama S, Weissman AM, Kuehn MR (2002) Identification of developmentally expressed proteins that functionally interact with Nedd4 ubiquitin ligase. J Biol Chem 277: 2897-2907

Nikko E, Marini AM, André B (2003) Permease recycling and ubiquitination status reveal a particular role for Bro1 in the multivesicular body pathway. J Biol Chem

Odorizzi G, Katzmann DJ, Babst M, Audhya A, Emr SD (2003) Bro1 is an endosomeassociated protein that functions in the MVB pathway in Saccharomyces cerevisiae. $J$ Cell Sci 116: 1893-1903

Omura F, Kodama Y, Ashikari T (2001a) The basal turnover of yeast branched-chain amino acid permease Bap2p requires its C-terminal tail. FEMS Microbiol Lett 194: 207-214

Omura F, Kodama Y, Ashikari T (2001b) The N-terminal domain of the yeast permease Bap2p plays a role in its degradation. Biochem Biophys Res Commun 287: 10451050

Ooi CE, Rabinovich E, Dancis A, Bonifacino JS, Klausner RD (1996) Copper-dependent degradation of the Saccharomyces cerevisiae plasma membrane copper transporter Ctr1p in the apparent absence of endocytosis. EMBO J 15: 3515-3523

Paddon C, Loayza D, Vangelista L, Solari R, Michaelis S (1996) Analysis of the localization of STE6/CFTR chimeras in a Saccharomyces cerevisiae model for the cystic fibrosis defect CFTR delta F508. Mol Microbiol 19: 1007-1017

Paiva S, Kruckeberg A, Casal M (2002) Utilization of green fluorescent protein as a marker for studying the expression and turnover of the monocarboxylate permease Jen $1 p$ of Saccharomyces cerevisiae. Biochem J 363: 737-744

Palmer LK, Wolfe D, Keeley JL, Keil RL (2002) Volatile anesthetics affect nutrient availability in yeast. Genetics 161: 563-574

Penalver E, Lucero P, Moreno E, Lagunas R (1999) Clathrin and two components of the COPII complex, Sec23p and Sec24p, could be involved in endocytosis of the Saccharomyces cerevisiae maltose transporter. J Bacteriol 181: 2555-2563

Peng J, Schwartz D, Elias JE, Thoreen CC, Cheng D, Marsischky G, Roelofs J, Finley D, Gygi SP (2003) A proteomics approach to understanding protein ubiquitination. Nat Biotechnol 21: 921-926

Petersson J, Pattison J, Kruckeberg AL, Berden JA, Persson BL (1999) Intracellular localization of an active green fluorescent protein-tagged Pho84 phosphate permease in Saccharomyces cerevisiae. FEBS Lett. 462: 37-42

Petris MJ, Mercer JF, Culvenor JG, Lockhart P, Gleeson PA, Camakaris J (1996) Ligandregulated transport of the Menkes copper P-type ATPase efflux pump from the Golgi apparatus to the plasma membrane: a novel mechanism of regulated trafficking. EMBO J 15: 6084-6095

Petris MJ, Smith K, Lee J, Thiele DJ (2003) Copper-stimulated endocytosis and degradation of the human copper transporter, hCtr1. J Biol Chem 278: 9639-96346

Petris MJ, Voskoboinik I, Cater M, Smith K, Kim BE, Llanos RM, Strausak D, Camakaris J, Mercer JF (2002) Copper-regulated trafficking of the Menkes disease copper ATPase is associated with formation of a phosphorylated catalytic intermediate. J Biol Chem 277: 46736-46742

Piper RC, Luzio JP (2001) Late endosomes: sorting and partitioning in multivesicular bodies. Traffic 2: 612-621.

Plant PJ, Lafont F, Lecat S, Verkade P, Simons K, Rotin D (2000) Apical membrane targeting of Nedd4 is mediated by an association of its C2 domain with annexin XIIlb. J Cell Biol 149: 1473-1484 
Plant PJ, Yeger H, Staub O, Howard P, Rotin D (1997) The C2 domain of the ubiquitin protein ligase Nedd4 mediates $\mathrm{Ca2+-}$ dependent plasma membrane localization. J Biol Chem 272: 32329-32336

Plemper RK, Egner R, Kuchler K, Wolf DH (1998) Endoplasmic reticulum degradation of a mutated ATP-binding cassette transporter Pdr5 proceeds in a concerted action of Sec61 and the proteasome. J Biol Chem 273: 32848-32856

Portnoy ME, Liu XF, Culotta VC (2000) Saccharomyces cerevisiae expresses three functionally distinct homologues of the nramp family of metal transporters. Mol Cell Biol 20: 7893-7902.

Raasi S, Pickart CM (2003) Rad23 ubiquitin-associated domains (UBA) inhibit 26 S proteasome-catalyzed proteolysis by sequestering lysine 48-linked polyubiquitin chains. J Biol Chem 278: 8951-8959

Ramamoorthy S, Blakely RD (1999) Phosphorylation and sequestration of serotonin transporters differentially modulated by psychostimulants. Science 285: 763-766

Riezman H (1998) Down regulation of yeast G protein-coupled receptors. Semin Cell Dev Biol 9: 129-134

Roberg KJ, Bickel S, Rowley N, Kaiser CA (1997a) Control of amino acid permease sorting in the late secretory pathway of Saccharomyces cerevisiae by SEC13, LST4, LST7 and LST8. Genetics 147: 1569-1584

Roberg KJ, Rowley N, Kaiser CA (1997b) Physiological regulation of membrane sorting late in the secretory pathway of Saccharomyces cerevisiae. J Cell Biol 137: 1469-1482

Roth AF, Davis NG (2000) Ubiquitination of the PEST-like endocytosis signal of the yeast afactor receptor. J Biol Chem 275: 8143-8153

Rotin D, Staub O, Haguenauer-Tsapis R (2000) Ubiquitination and endocytosis of plasma membrane proteins: role of Nedd4/Rsp5p family of ubiquitin-protein ligases [In Process Citation]. J Membr Biol 176: 1-17

Royle SJ, Murrell-Lagnado RD (2003) Constitutive cycling: a general mechanism to regulate cell surface proteins. Bioessays 25: 39-46

Saunders C, Ferrer JV, Shi L, Chen J, Merrill G, Lamb ME, Leeb-Lundberg LM, Carvelli L, Javitch JA, Galli A (2000) Amphetamine-induced loss of human dopamine transporter activity: an internalization-dependent and cocaine-sensitive mechanism. Proc Natl Acad Sci U S A 97: 6850-6855

Schild L, Lu Y, Gautschi I, Schneeberger E, Lifton RP, Rossier BC (1996) Identification of a PY motif in the epithelial $\mathrm{Na}$ channel subunits as a target sequence for mutations causing channel activation found in Liddle syndrome. EMBO J 15: 2381-2387

Schmidt A, Beck T, Koller A, Kunz J, Hall M (1998) The TOR nutrient signalling pathway phosphorylates NPR1 and inhibits turnover of the tryptophan permease. EMBO J 17: 6924-6931

Schwake M, Friedrich T, Jentsch TJ (2001) An internalization signal in CIC-5, an endosomal $\mathrm{Cl}$-channel mutated in dent's disease. J Biol Chem 276: 12049-12054

Séron K, Blondel M-O, Haguenauer-Tsapis R, Volland C (1999) Uracil-induced down regulation of the yeast uracil permease. J Bacteriol 181: 1793-1800

Shcherbik N, Zoladek T, Nickels JT, Haines DS (2003) Rsp5p is required for ER bound Mga2p120 polyubiquitination and release of the processed/tethered transactivator Mga2p90. Curr Biol 13: 1227-1233

Shih SC, Katzmann DJ, Schnell JD, Sutanto M, Emr SD, Hicke L (2002) Epsins and Vps27p/Hrs contain ubiquitin-binding domains that function in receptor endocytosis. Nat Cell Biol 4: 389-393.

Shih SC, Sloper-Mould KE, Hicke L (2000) Monoubiquitin carries a novel internalization signal that is appended to activated receptors. EMBO J 19: 187-198

Silve S, Volland C, Garnier C, Jund R, Chevallier MR, Haguenauer-Tsapis R (1991) Membrane insertion of uracil permease, a polytopic yeast plasma membrane protein. Mol Cell Biol 11: 1114-1124 
Siniossoglou S, Pelham HR (2001) An effector of Ypt6p binds the SNARE Tlg1p and mediates selective fusion of vesicles with late Golgi membranes. EMBO J 20: 59915998

Soetens O, De Craene JO, André B (2001) Ubiquitin is required for sorting to the vacuole of the yeast general amino acid permease, Gap1. J Biol Chem 276: 43949-43957.

Springael J-Y, De Craene J-O, André B (1999a) The yeast Npi1/Rsp5 ubiquitin-ligase lacking its $\mathrm{N}$-terminal $\mathrm{C} 2$ domain is competent for ubiquitination but not for subsequent endocytosis of the Gap1 permease. Biochem Biophys Res Comm 257: 561-566

Springael J-Y, Galan J-M, Haguenauer-Tsapis R, André B (1999b) NH4+-induced downregulation of the Saccharomyces cerevisiae Gap1p permease involves its ubiquitination with lysine-63-linked chains. J Cell Sci 112: 1375-1383

Springael JY, André B (1998) Nitrogen-regulated ubiquitination of the Gap1 permease of Saccharomyces cerevisiae. Mol Biol Cell 9: 1253-1263.

Springael JY, Nikko E, André B, Marini AM (2002) Yeast Npi3/Bro1 is involved in ubiquitindependent control of permease trafficking. FEBS Lett 517: 103-109

Stanbrough M, Magasanik B (1995) Transcriptional and posttranslational regulation of the general amino acid permease of Saccharomyces cerevisiae. J Bacteriol 177: 94-102

Staub O, Gautschi I, Ishikawa T, Breitschop K, Ciechanover A, Schild L, Rotin D (1997) Regulation of stability and function of the epithelial $\mathrm{Na}+$ channel (ENaC) by ubiquitination. EMBO J 16: 6325-6336

Swaminathan S, Amerik AY, Hochstrasser M (1999) The Doa4 deubiquitinating enzyme is required for ubiquitin homeostasis in yeast. Mol Biol Cell 10: 2583-2594

Swanson R, Locher M, Hochstrasser M (2001) A conserved ubiquitin ligase of the nuclear envelope/endoplasmic reticulum that functions in both ER-associated and Matalpha2 repressor degradation. Genes Dev 15: 2660-2674.

Szaszi K, Paulsen A, Szabo EZ, Numata M, Grinstein S, Orlowski J (2002) Clathrinmediated endocytosis and recycling of the neuron-specific $\mathrm{Na}+\mathrm{H}+$ exchanger NHE5 isoform. Regulation by phosphatidylinositol 3'-kinase and the actin cytoskeleton. $\mathrm{J}$ Biol Chem 277: 42623-42632

Terrell J, Shih S, Dunn R, Hicke L (1998) A function for monoubiquitination in the internalization of a $\mathrm{G}$ protein-coupled receptor. Mol Cell 1: 193-202

Touret N, Furuya W, Forbes J, Gros P, Grinstein S (2003) Dynamic traffic through the recycling compartment couples the metal transporter Nramp2 (DMT1) with the transferrin receptor. J Biol Chem 278: 25548-25557

Tschopp JF, Emr SD, Field C, Schekman R (1986) GAL2 codes for a membrane-bound subunit of the galactose permease in Saccharomyces cerevisiæ. J Bacteriol 166: 313-318

Umebayashi K, Nakano A (2003) Ergosterol is required for targeting of tryptophan permease to the yeast plasma membrane. J Cell Biol 161: 1117-11131

Urban-Grimal D, Pinson B, Chevallier J, Haguenauer-Tsapis R (1995) Replacement of Lys by Glu in a transmembrane segment strongly impairs the function of the uracil permease from Saccharomyces cerevisiae. Biochem J 308: 847-851

Urbanowski J, Piper RC (2001) Ubiquitin sorts proteins into the lumenal degradative compartment of the late endosome/vacuole. Traffic 2: 622-630

Van Belle D, André B (2001) A genomic view of yeast membrane transporters. Curr Opin Cell Biol 13: 389-398

Vandenbol M, Jauniaux JC, Grenson M (1990) The Saccharomyces cerevisiae NPR1 gene required for the activity of ammonia-sensitive amino acid permeases encodes a protein kinase homologue. Mol Gen Genet 222: 393-399

Vandenbol M, Jauniaux JC, Vissers S, Grenson M (1987) Isolation of the NPR1 gene responsible for the reactivation of ammonia-sensitive amino-acid permeases in 
Saccharomyces cerevisiae. RNA analysis and gene dosage effects. Eur J Biochem 164: $607-612$

Vecchione A, Marchese A, Henry P, Rotin D, Morrione A (2003) The Grb10/Nedd4 complex regulates ligand-induced ubiquitination and stability of the insulin-like growth factor I receptor. Mol Cell Biol 23: 3363-3372

Volland C, Garnier C, Haguenauer-Tsapis R (1992) In vivo phosphorylation of the yeast uracil permease. J Biol Chem 267: 23767-23771

Volland C, Urban-Grimal D, Géraud G, Haguenauer-Tsapis R (1994) Endocytosis and degradation of the yeast uracil permease under adverse conditions. J Biol Chem 269: 9833-9841

Wang G, McCaffery JM, Wendland B, Dupre S, Haguenauer-Tsapis R, Huibregtse JM (2001) Localization of the Rsp5p ubiquitin-protein ligase at multiple sites within the endocytic pathway. Mol Cell Biol 21: 3564-3575.

Wang G, Yang J, Huibregste J (1999) Fonctional Domains of the RSP5 Ubiquitin-Protein ligase. Mol Cell Biol 19: 342-352

Warner JR (1999) The economics of ribosome biosynthesis in yeast. Trends Biochem Sci 24: 437-440

Weissman AM (2001) Themes and variations on ubiquitylation. Nat Rev Mol Cell Biol 2: 169178.

Wells KM, Rao R (2001) The yeast $\mathrm{Na}+/ \mathrm{H}+$ exchanger $\mathrm{Nhx1}$ is an $\mathrm{N}$-linked glycoprotein. Topological implications. J Biol Chem 276: 3401-3407

Welsch CA, Hagiwara S, Goetschy JF, Movva NR (2003) Ubiquitin pathway proteins influence the mechanism of action of the novel immunosuppressive drug FTY720 in Saccharomyces cerevisiae. J Biol Chem 278: 26976-26982

Wendell DL, Bisson LF (1993) Physiological characterization of putative high-affinity glucose transport protein Hxt2 of Saccharomyces cerevisiae by use of anti-synthetic peptide antibodies. J Bacteriol 175: 7689-7696

Wendland B (2002) Epsins: adaptors in endocytosis? Nat Rev Mol Cell Biol 3: 971-977

Wendland B, Steece KE, Emr SD (1999) Yeast epsins contain an essential N-terminal ENTH domain, bind clathrin and are required for endocytosis. EMBO J 18: 4383-43893

Whitworth TL, Quick MW (2001) Substrate-induced regulation of gamma-aminobutyric acid transporter trafficking requires tyrosine phosphorylation. J Biol Chem 276: 4293242937

Yashiroda H, Kaida D, Toh-e A, Kikuchi Y (1998) The PY-motif of Bul1 protein is essential for growth of Saccharomyces cerevisiae under various stress conditions. Gene 225: $39-46$

Yashiroda H, Oguchi T, Yasuda Y, Toh-e A, Kikuchi Y (1996) Bul1, a new protein that bind to the Rsp5 Ubiquitin ligase. Mol Cell Biol 16: 3255-3263

Yonkovich J, McKenndry R, Shi X, Zhu Z (2002) Copper ion-sensing transcription factor Mac1p post-translationally controls the degradation of its target gene product Ctr1p. J Biol Chem 277: 23981-23984

Zhao R, Reithmeier RA (2001) Expression and characterization of the anion transporter homologue YNL275w in Saccharomyces cerevisiae. Am J Physiol Cell Physiol 281: C33-45 


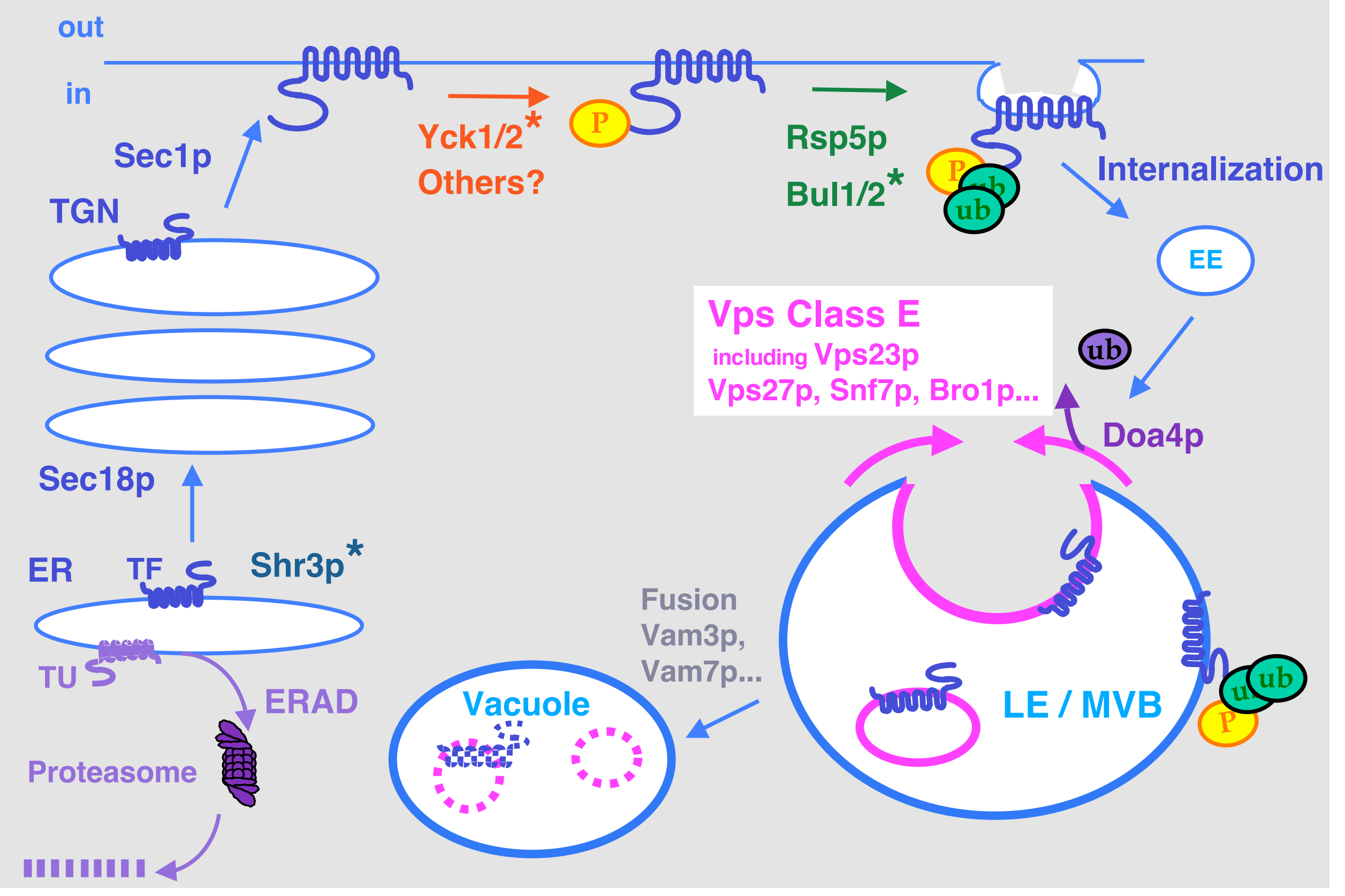


Type of ubiquitylation of yeast transporters

\section{UBIQUITIN}

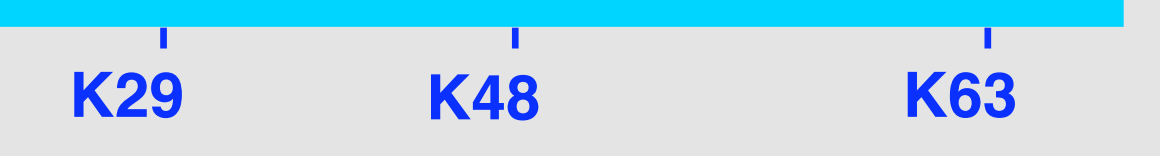

\section{POLYUBIQUITYLATION}

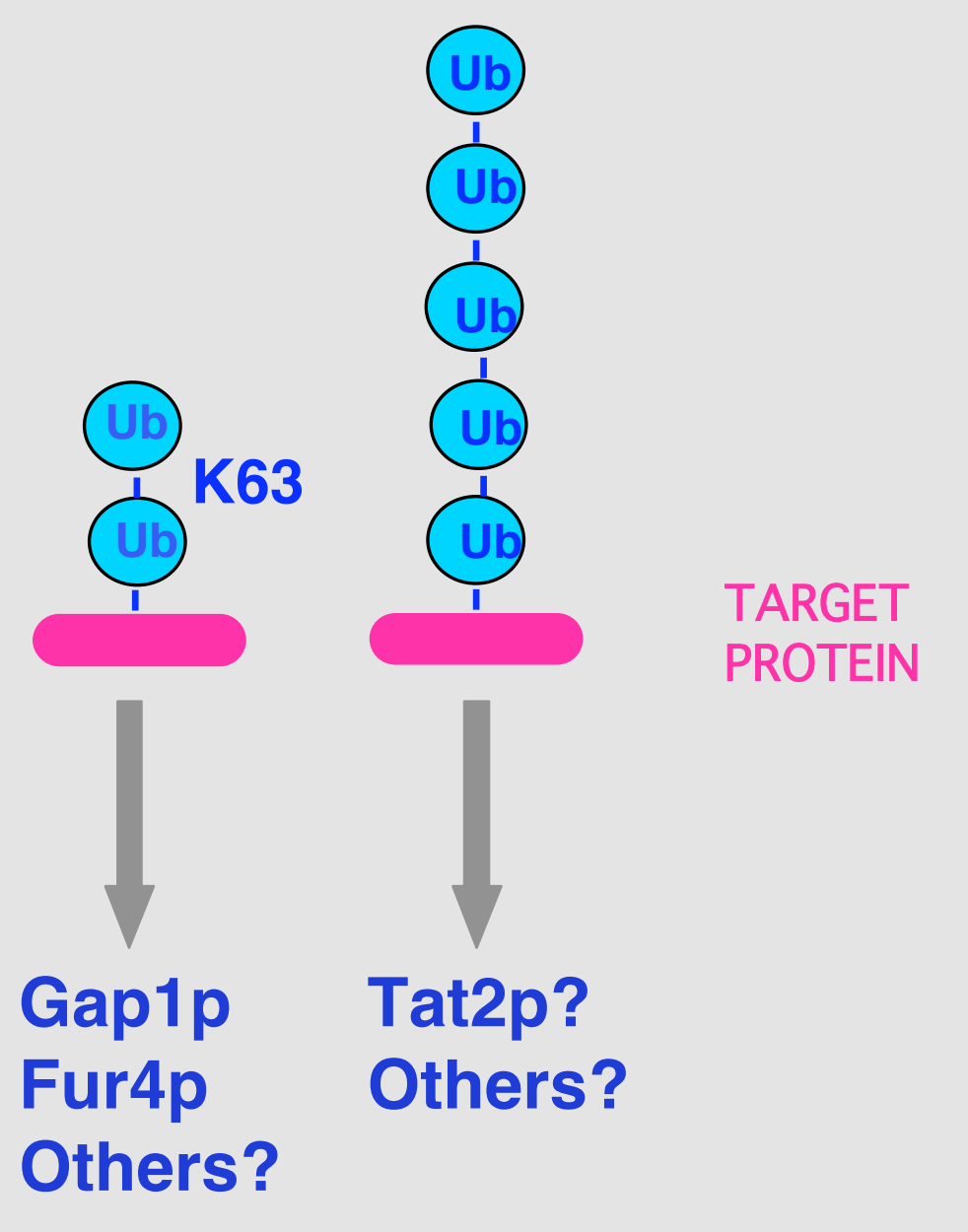

PROTEASOME

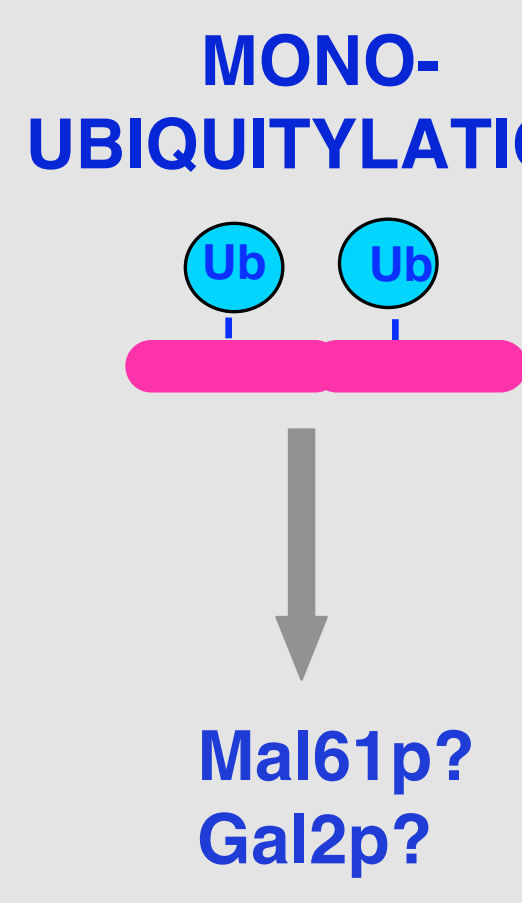


WT

Fur4-GFP GFP

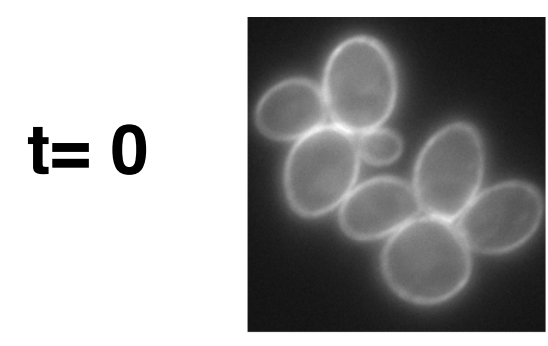

KRFur4-GFP

GFP CMAC

$t=2 h$
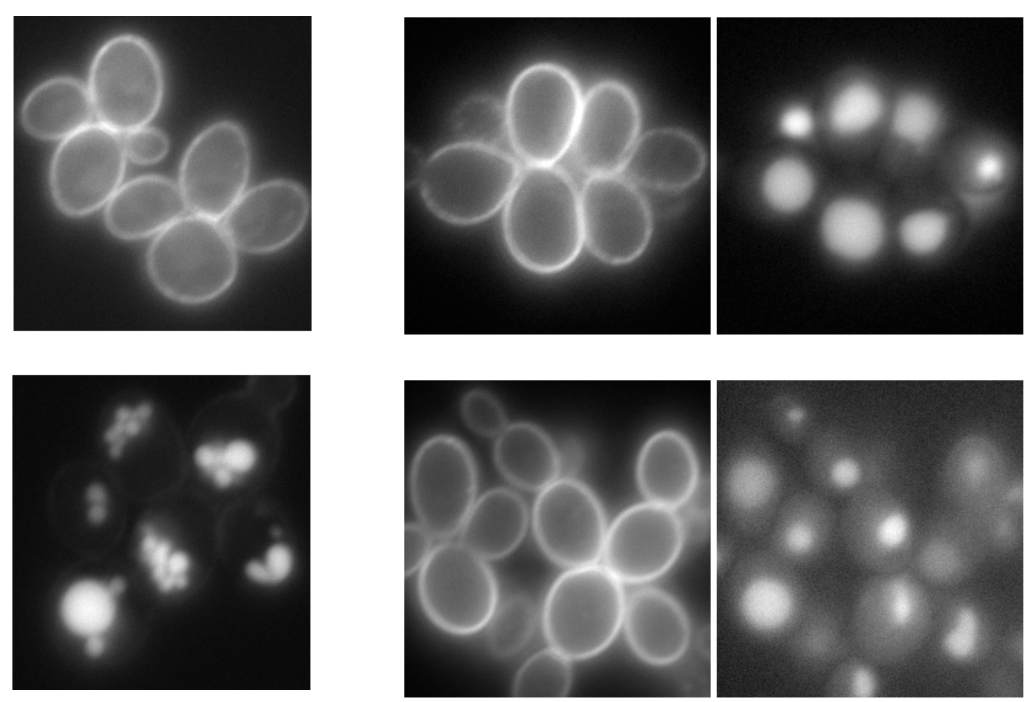

Uracil induced

Fur4-GFP endocytosis

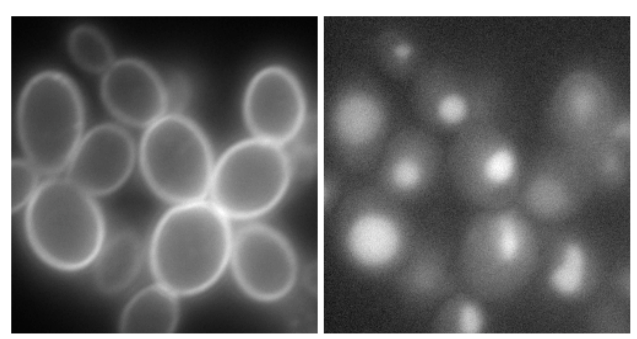

npi1 / rsp5

Fur4-GFP

Ub-KRFur4-GFP

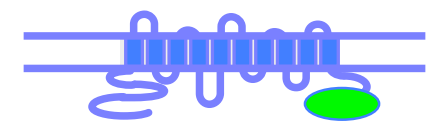

Fur4

GFP

or

$\mathbf{t}=\mathbf{0}$

KRFur4

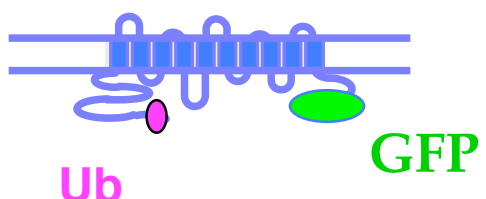

Ub

GFP
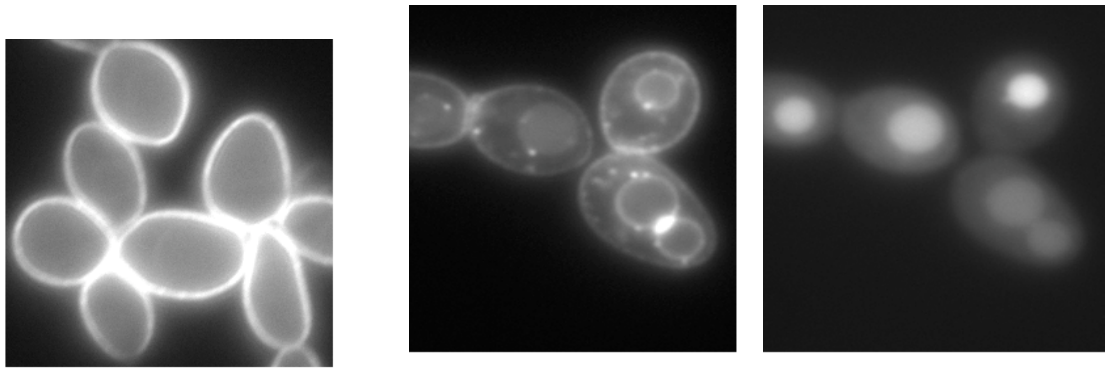

$t=2 h$
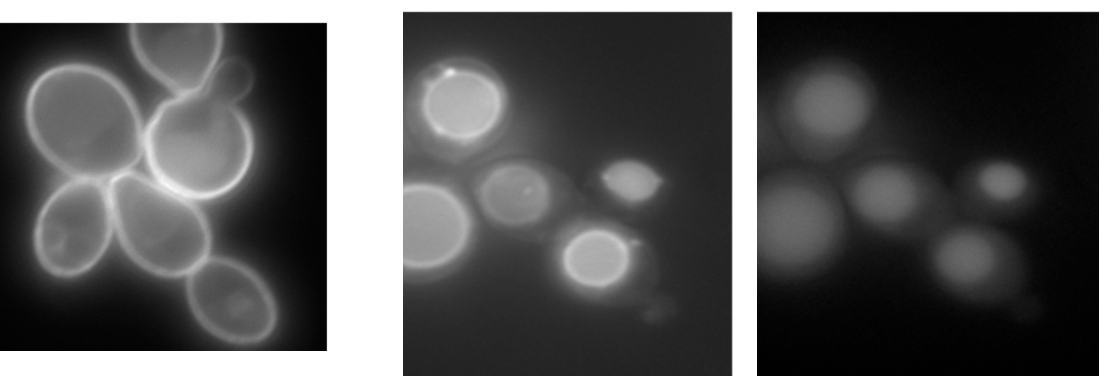


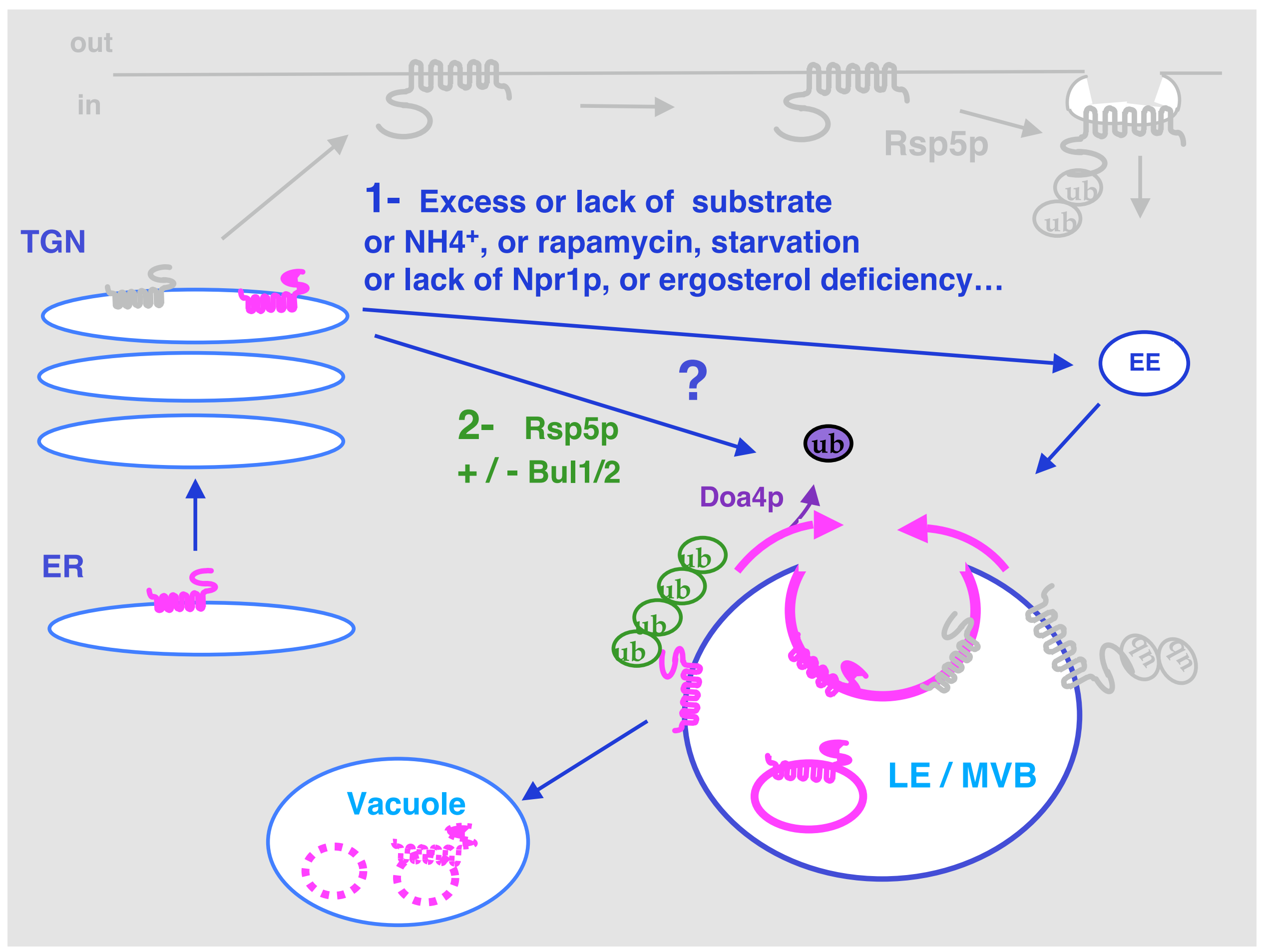




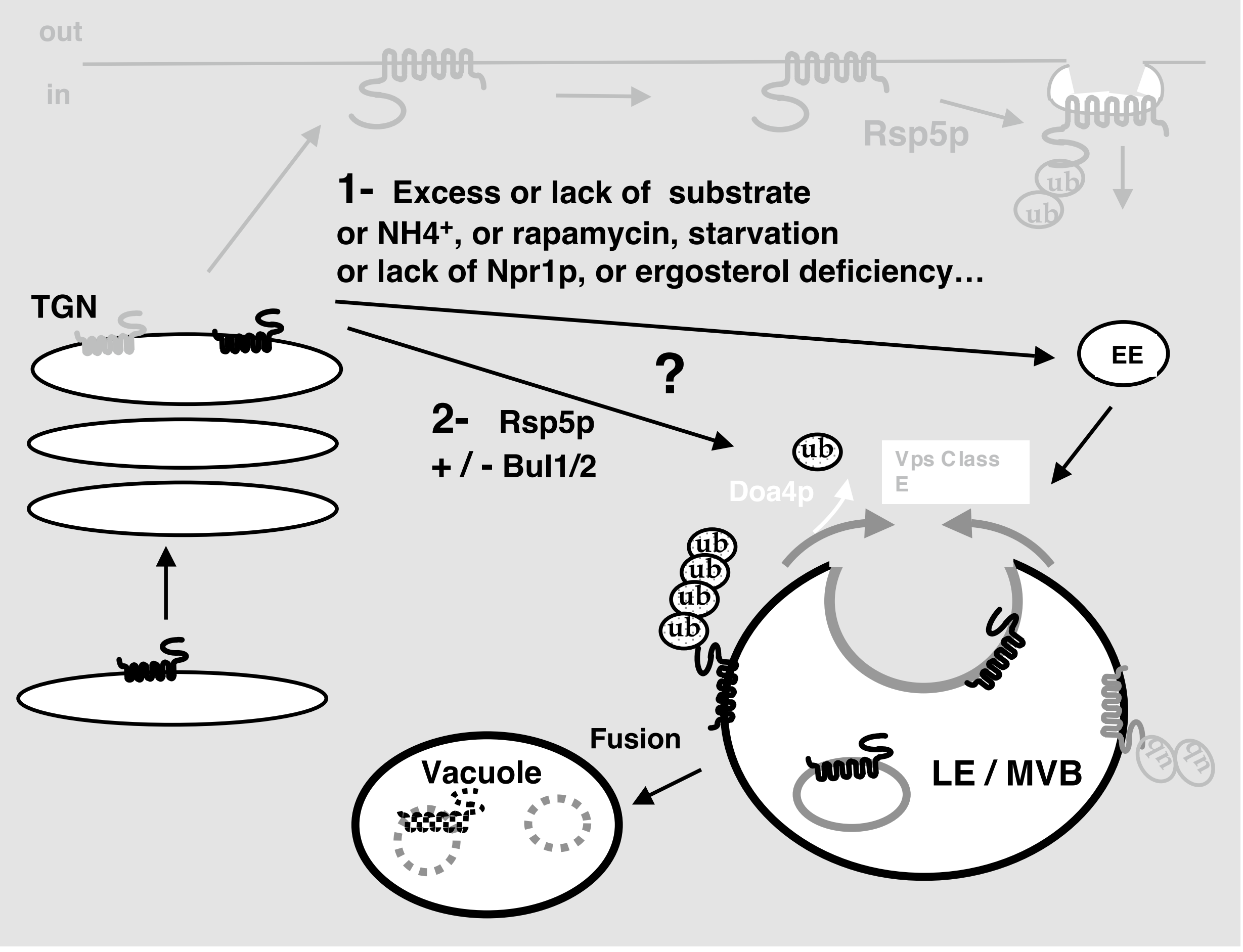

